\title{
ALGEBRO-GEOMETRIC VERSION OF NEVANLINNA'S LEMMA ON LOGARITHMIC DERIVATIVE AND APPLICATIONS
}

\author{
KATSUTOSHI YAMANOI
}

\begin{abstract}
In this paper we shall establish some generalization of Nevanlinna's Lemma on Logarithmic Derivative to the case of meromorphic maps from a finite analytic covering space over the $m$-dimensional complex affine space $\mathbb{C}^{m}$ to a smooth complex projective variety. Then we shall apply this to "the Second Main Theorem" in Nevanlinna theory in several complex variables.
\end{abstract}

\section{$\S 1$. Introduction}

In 1925, R. Nevanlinna [Nev39] proved what is known as the lemma of the logarithmic derivative for a meromorphic function $f$ defined on the complex plane $\mathbb{C}$. This lemma was the main technical tool in the proof of his celebrated Second Main Theorem for a meromorphic function $f$, and it has served as a similar function in much of the subsequent work in the value distribution theory. After Nevanlinna's work, A. L. Vitter [Vi77] generalized this lemma to a meromorphic function on the $m$-dimensional complex affine space $\mathbb{C}^{m}$.

Since a meromorphic function $f$ on $\mathbb{C}^{m}$ can be considered as a meromorphic map $f: \mathbb{C}^{m} \rightarrow \mathbb{P}^{1}$, it is a natural problem to generalize the lemma of the logarithmic derivative to the case of a meromorphic map $f: \mathbb{C}^{m} \rightarrow X$ for a complex projective manifold $X$. In this direction, J. Noguchi [No77], [No85] established the lemma of the logarithmic derivative for a meromorphic map $f: Y \rightarrow X$ and global logarithmic 1-forms on $X$, where $Y \stackrel{\pi}{\rightarrow} \mathbb{C}^{m}$ is a finite analytic covering space over $\mathbb{C}^{m}$. And he established the second main theorem type inequality for a meromorphic map $f: Y \rightarrow X$ and a divisor $D \subset X$ on $X$ such that there are sufficiently many global logarithmic 1-forms on $X$ with only logarithmic poles on $D$.

The main purpose of this paper is to establish another geometric formulation of the lemma of the logarithmic derivative for a meromorphic map

Received September 14, 2000.

2000 Mathematics Subject Classification: 32H30. 
$f: Y \rightarrow X$ using the jet bundle over $X$ and the jet lifting of $f$ (Theorem 3.2.1). In the case $X=\mathbb{P}^{1}$, after some observations, one sees that this formulation is equivalent to the classical Nevanlinna, Vitter type analytic formulation (see Remark 3.2.5). As far as the author knows, this geometric formulation was first discussed by R. Kobayashi [Kob91a]-[Kob00] in the context of his very interesting idea of "Radon transform" and "integral geometry associated to it". We shall call this geometric formulation Algebro-Geometric Version of Nevanlinna's Lemma on Logarithmic Derivative (ANLD) after [Kob96a]. Kobayashi [Kob91a], [Kob00] proved this ANLD when $X$ is an Abelian variety and using this, he proved the second main theorem for holomorphic curves in Abelian varieties. Kobayashi [Kob96a], [Kob96b] also give a sketch of a proof of ANLD for a general $X$ under some non-degeneracy condition on $f$ and show a plan to approach the second main theorem for holomorphic curves into $X$ using ANLD. But at present, as far as the author knows, no detail can be found for these discussions concerning a general $X$. In this paper, we shall give a simpler and direct proof of ANLD for a general $X$ together with some more generalization of [Kob96a], [Kob96b]. In consequence of our simple proof, we can prove ANLD without non-degeneracy conditions on $f$ and our ANLD gives sharp estimates.

After giving these technical improvements, we shall discuss some applications of our results to the second main theorem in Nevanlinna theory in several complex variables. The first application is equidimensional value distribution theory. This theory was established by J. Carlson-P. Griffiths [CG72], P. Griffiths-J. King [GK73], B. Shiffman [Sh75], J. Noguchi [No76]. Our argument gives a direct connection between the lemma of the logarithmic derivative and the second main theorem for the equidimensional case. The second application is the second main theorem for holomorphic curves in Abelian varieties. This theory was established by Kobayashi [Kob00] and J. Noguchi-J. Winkelmann-K. Yamanoi [NoWY02]. (The paper [NoWY02] also treats the semi-Abelian cases. See also Section 5 in this paper.) Here, we apply ANLD to obtain the second main theorem for holomorphic curves in Abelian varieties, following Kobayashi's argument in [Kob91a], [Kob00]. The third application is the second main theorem for hyperplanes in the projective space $\mathbb{P}^{n}$. This theory was due to H. Cartan [C33] and our method is very similar to Cartan's. We consider some lifting of holomorphic curves to the anti-canonical bundle of projective space $\mathbb{P}^{n}$, while Cartan considered the Wronskian. In these applications, there is a 
somewhat common algebro-geometric structure in the process of deriving the second main theorem from ANLD. We give a sufficient condition for the second main theorem for holomorphic curves in a projective manifold $X$ (Proposition 4.2.1). This condition is an algebro-geometric condition of the structure of the jet space over $X$, and we shall show the second and the third applications above as consequences of the condition. In the case of equidimensional value distribution theory, we may say that the condition is 'always satisfied', in some sense. Our condition in Proposition 4.2.1 is also very closely related to the frame-work of negative curvature method in hyperbolicity problems, which was first discussed by M. Green-P. Griffiths [GG80] (Corollary 4.2.3).

Contents of this paper are the following. In Section 2, we shall review notations and well known results in Nevanlinna theory. Since natural compactifications of jet spaces have quotient singularities, we have to prepare some notations in Nevanlinna theory for singular varieties. In this section, we shall also define proximity functions with respect to arbitrary closed subschemes. We need the proximity functions with respect to higher codimensional subvarieties for the statement of ANLD. In Section 3, we shall give a proof of ANLD for a general $X$. The essential point is Proposition 3.1.1 which gives estimates of Weil functions. In Section 4, we discuss the second main theorem in the above cases. In Section 5, we shall extend our ANLD to the case of logarithmic jet spaces, and apply this to holomorphic curves in semi-Abelian varieties to obtain the second main theorem.

I wish to express my sincere gratitudue to Professor R. Kobayashi. His beautiful papers and ideas got me started in the subject and inspired me. I warmly thank Professor J. Noguchi for many helpful suggestions which helped me to improve these notes and simplify a proof of the main result. The result of this paper is authors doctoral thesis at RIMS, Kyoto University, March 2000, and I am deeply indebted to my thesis advisor Professor Y. Ihara for his continuous and warm encouragements.

\section{§2. Preliminaries}

2.1. In this paper, all varieties are irreducible algebraic varieties defined over $\mathbb{C}$. Let $V$ be a quasi-projective variety. Let $\mathcal{C}(V)$ be the set of all $\mathbb{R}$-valued continuous functions (with respect to the usual Hausdorff topology) which are defined on some Zariski open sets of $V$. More precisely, 
$\mathcal{C}(V)$ is the germ of the sheaf

$\left\{\begin{array}{l|l}U & \begin{array}{l}U ; \text { Zariski open } \\ \text { subset of } V\end{array}\end{array}\right\} \longmapsto\left\{\begin{array}{l|l}g \mid \begin{array}{l}g: U \rightarrow \mathbb{R} \text {; continuous function with } \\ \text { respect to the usual Hausdorff topology }\end{array}\end{array}\right\}$ at the "generic point" of $V$ (note that $V$ is irreducible).

Definition 2.1.1. Let $V$ be a quasi-projective variety. Let $g, h \in$ $\mathcal{C}(V)$. We shall write $g \leq_{\langle V\rangle} h$ if there is a continuous function $\alpha$ which is defined on $V$ and satisfies the condition $g \leq h+\alpha$ on some non-empty Zariski open set where $g$ and $h$ are both defined. We shall write $g \equiv\langle V\rangle h$ if $g \leq_{\langle V\rangle} h$ and $h \leq_{\langle V\rangle} g$.

The relation $\equiv\langle V\rangle$ defines the equivalent relation on the set $\mathcal{C}(V)$, and $\leq\langle V\rangle$ defines the order relation on the set $\mathcal{C}(V) / \equiv\langle V\rangle$. Let $g, h \in \mathcal{C}(V)$. Then $\min (g, h)$ defines the continuous function on some Zariski open set of $V$ where $g$ and $h$ are both defined. Hence $\min (g, h) \in \mathcal{C}(V)$.

Lemma 2.1.2. (1) Let $g, g^{\prime}, h, h^{\prime} \in \mathcal{C}(V)$, and let $g \equiv\langle V\rangle g^{\prime}, h \equiv\langle V\rangle h^{\prime}$. Then

$$
\min (g, h) \equiv\langle V\rangle \min \left(g^{\prime}, h^{\prime}\right) .
$$

(2) Let $\left\{U_{\epsilon}\right\}_{\epsilon \in \Upsilon}$ be a Zariski open covering of $V$. Let $g, h \in \mathcal{C}(V)$. Then

$$
g \leq\langle V\rangle h \Longleftrightarrow g \leq_{\left\langle U_{\epsilon}\right\rangle} h \quad \text { for all } \epsilon \in \Upsilon .
$$

Proof. (1) By our assumptions $g \equiv\langle V\rangle g^{\prime}, h \equiv\langle V\rangle h^{\prime}$, there are a nonempty Zariski open set $U \subset V$ and a continuous function $\alpha$ on $V$ satisfying the condition

$$
\left|g-g^{\prime}\right| \leq \alpha \text { and }\left|h-h^{\prime}\right| \leq \alpha \text { on } U
$$

By $g \leq g^{\prime}+\alpha$ and $h \leq h^{\prime}+\alpha$, we have $\min (g, h) \leq \min \left(g^{\prime}, h^{\prime}\right)+\alpha$ on $U$, and by $g^{\prime} \leq g+\alpha$ and $h^{\prime} \leq h+\alpha$, we have $\min \left(g^{\prime}, h^{\prime}\right) \leq \min (g, h)+\alpha$ on $U$. Hence we have $\min (g, h) \equiv\langle V\rangle \min \left(g^{\prime}, h^{\prime}\right)$.

(2) The part $\Longrightarrow$ is obvious, so we shall prove the part $\Longleftarrow$. Since $V$ is Noetherian, we can take a finite subset $\Upsilon^{\prime}$ of $\Upsilon$ such that $\left\{U_{\epsilon}\right\}_{\epsilon \in \Upsilon^{\prime}}$ is an open covering of $V$. Then by our assumption $g \leq_{\left\langle U_{\epsilon}\right\rangle} h$, there is a continuous function $\alpha_{\epsilon}$ on $U_{\epsilon}$ such that $g \leq h+\alpha_{\epsilon}$ on some Zariski open subset $U_{\epsilon}^{\prime}$ of $U_{\epsilon}$. Let $\left\{\rho_{\epsilon}\right\}_{\epsilon \in \Upsilon^{\prime}}$ be a partition of unity on $V$ consists of an open covering $\left\{U_{\epsilon}\right\}_{\epsilon \in \Upsilon^{\prime}}$. Put $\alpha=\sum_{\epsilon \in \Upsilon^{\prime}} \alpha_{\epsilon} \rho_{\epsilon}$. Then $\alpha$ is a continuous function on $V$, and we have $g \leq h+\alpha$ on $\bigcup_{\epsilon \in \Upsilon^{\prime}} U_{\epsilon}^{\prime}$. Hence we have $g \leq\langle V\rangle$. 
2.2. Let $V$ be a quasi-projective variety over $\mathbb{C}$. Let $Z \subset V$ be a closed subscheme. We shall define a Weil function $\lambda_{Z}$ for $Z$ to be a continuous function $\lambda_{Z}: V-\operatorname{supp} Z \rightarrow \mathbb{R}$ satisfying a certain condition described below. Here $\operatorname{supp} Z$ is the support of $Z$. Note that the closed subschemes $Z \subset V$ are in one-to-one correspondence with the ideal sheaves $\mathcal{I}_{Z} \subset \mathcal{O}_{V}$. We will often implicitly identify a subscheme $Z$ with its ideal sheaf $\mathcal{I}_{Z}$.

Definition 2.2.1. Let $Z \subset V$ be a closed subscheme. A Weil function $\lambda_{Z}$ for $Z$ is a continuous function $\lambda_{Z}: V-\operatorname{supp} Z \rightarrow \mathbb{R}$ satisfying the condition that there exist an affine Zariski open covering $\left\{U_{\epsilon}\right\}_{\epsilon \in \Upsilon}$ of $V$ and a system of generators $f_{1}^{\epsilon}, \ldots, f_{r_{\epsilon}}^{\epsilon} \in \Gamma\left(U_{\epsilon}, \mathcal{O}_{U_{\epsilon}}\right)$ of the ideal $\Gamma\left(U_{\epsilon}, \mathcal{I}_{Z}\right) \subset$ $\Gamma\left(U_{\epsilon}, \mathcal{O}_{U_{\epsilon}}\right)$ such that

$$
\lambda_{Z}(P) \equiv\left\langle U_{\epsilon}\right\rangle-\log \max _{1 \leq i \leq r_{\epsilon}}\left|f_{i}^{\epsilon}(P)\right|
$$

Remark 2.2.2. (1) Let $D \subset V$ be an effective Cartier divisor on $V$. Then $D$ is naturally equipped with the closed subscheme structure (because $D$ has local equations), and we shall define $\lambda_{D}$ with respect to this closed subscheme structure. More generally, if $D$ is a $\mathbb{Q}$-Cartier divisor and $n D$ is a Cartier divisor, then we shall define $\lambda_{D}$ to be $\frac{1}{n} \lambda_{n D}$ (this definition is well-defined, cf. Lemma 2.2 .9 (2) below).

(2) Let $V$ be non-singular. Let $D \subset V$ be an effective divisor on $V$ and take a Hermitian metric $\|\cdot\|$ for the corresponding line bundle $\mathcal{O}(D)$. Let $\sigma$ be a canonical section of $\mathcal{O}(D)$ uniquely determined up to a constant multiple by the condition that the zero divisor of $\sigma$ coincides with $D$. Then the function $\lambda_{D}(P)=-\log \|\sigma(P)\|$ is a Weil function for $D$.

Proposition 2.2.3. Let $V$ be a quasi-projective variety, and let $Z$ be a closed subscheme of $V$. Then we have the followings.

(1) A Weil function $\lambda_{Z}$ exists for every closed subscheme $Z \subset V$.

(2) Let $\lambda_{Z}$ and $\lambda_{Z}^{\prime}$ be two Weil functions for $Z$. Then $\lambda_{Z} \equiv\langle V\rangle \lambda_{Z}^{\prime}$. If $V$ is projective, then the difference of two Weil functions for $Z$ is bounded, i.e. Weil function for $Z$ is unique up to $O(1)$.

(3) Let $\lambda_{Z}$ be a Weil function for $Z$. Then for every affine Zariski open set $W \subset V$ and every system of generators $g_{1}, \ldots, g_{s} \in \Gamma\left(W, \mathcal{O}_{W}\right)$ of the ideal $\Gamma\left(W, \mathcal{I}_{Z}\right) \subset \Gamma\left(W, \mathcal{O}_{W}\right)$, we have

$$
\lambda_{Z}(P) \equiv\langle W\rangle-\log \max _{1 \leq i \leq s}\left|g_{i}(P)\right| .
$$


Proof of (3). Since $\lambda_{Z}$ is a Weil function for $Z$, we can take an affine open covering $\left\{U_{\epsilon}\right\}_{\epsilon \in \Upsilon}$ of $V$ such that for each $U_{\epsilon}$, there is a system of generators $f_{1}^{\epsilon}, \ldots, f_{r_{\epsilon}}^{\epsilon} \in \Gamma\left(U_{\epsilon}, \mathcal{O}_{U_{\epsilon}}\right)$ of the ideal $\Gamma\left(U_{\epsilon}, \mathcal{I}_{Z}\right) \subset \Gamma\left(U_{\epsilon}, \mathcal{O}_{U_{\epsilon}}\right)$ such that

$$
\lambda_{Z}(P) \equiv\left\langle U_{\epsilon}\right\rangle-\log \max _{1 \leq i \leq r_{\epsilon}}\left|f_{i}^{\epsilon}(P)\right| .
$$

Set $W_{\epsilon}=W \cap U_{\epsilon}$. Then by Lemma $2.1 .2(2)$, it suffices to show

$$
\lambda_{Z}(P) \equiv\left\langle W_{\epsilon}\right\rangle-\log \max _{1 \leq j \leq s}\left|g_{j}(P)\right| .
$$

Since $f_{1}^{\epsilon}, \ldots, f_{r_{\epsilon}}^{\epsilon} \in \Gamma\left(W_{\epsilon}, \mathcal{O}_{W_{\epsilon}}\right)$ and $g_{1}, \ldots, g_{s} \in \Gamma\left(W_{\epsilon}, \mathcal{O}_{W_{\epsilon}}\right)$ are two systems of generators of the ideal $\Gamma\left(W_{\epsilon}, \mathcal{I}_{Z}\right) \subset \Gamma\left(W_{\epsilon}, \mathcal{O}_{W_{\epsilon}}\right)$, there exist $h_{i}^{j} \in$ $\Gamma\left(W_{\epsilon}, \mathcal{O}_{W_{\epsilon}}\right)$ for $1 \leq j \leq s, 1 \leq i \leq r_{\epsilon}$ such that $g_{j}=\sum_{i=1}^{r_{\epsilon}} h_{i}^{j} f_{i}^{\epsilon}$. Then we have

$$
\left|g_{j}(P)\right| \leq \sum_{i=1}^{r_{\epsilon}}\left|h_{i}^{j}(P)\right|\left|f_{i}^{\epsilon}(P)\right| \leq\left(\sum_{i=1}^{r_{\epsilon}}\left|h_{i}^{j}(P)\right|\right) \max _{1 \leq i \leq r_{\epsilon}}\left|f_{i}^{\epsilon}(P)\right| .
$$

Hence we have

$$
\begin{aligned}
\max _{1 \leq i \leq r_{\epsilon}}\left|f_{i}^{\epsilon}(P)\right| & \leq \max _{\substack{1 \leq i \leq r_{\epsilon} \\
1 \leq j \leq s}}\left\{\left|f_{i}^{\epsilon}(P)\right|,\left|g_{j}(P)\right|\right\} \\
& \leq \max _{1 \leq j \leq s}\left\{1,\left(\sum_{i=1}^{r_{\epsilon}}\left|h_{i}^{j}(P)\right|\right)\right\} \max _{1 \leq i \leq r_{\epsilon}}\left|f_{i}^{\epsilon}(P)\right| .
\end{aligned}
$$

Put $\gamma_{\epsilon}=\log \max _{1 \leq j \leq s}\left\{1,\left(\sum_{i=1}^{r_{\epsilon}}\left|h_{i}^{j}\right|\right)\right\}$. Then $\gamma_{\epsilon}$ is a continuous function on $W_{\epsilon}$ and we have

$$
\left|\log \max _{\substack{1 \leq i \leq r_{\epsilon} \\ 1 \leq j \leq s}}\left\{\left|f_{i}^{\epsilon}(P)\right|,\left|g_{j}(P)\right|\right\}-\log \max _{1 \leq i \leq r_{\epsilon}}\right| f_{i}^{\epsilon}(P)|| \leq \gamma_{\epsilon}(P) .
$$

Hence we have

$$
\log \max _{\substack{1 \leq i \leq r_{\epsilon} \\ 1 \leq j \leq s}}\left\{\left|f_{i}^{\epsilon}(P)\right|,\left|g_{j}(P)\right|\right\} \equiv\left\langle W_{\epsilon}\right\rangle \log \max _{1 \leq i \leq r_{\epsilon}}\left|f_{i}^{\epsilon}(P)\right| .
$$

By the same argument, we have

$$
\log \max _{1 \leq i \leq r_{\epsilon}}\left\{\left|f_{i}^{\epsilon}(P)\right|,\left|g_{j}(P)\right|\right\} \equiv\left\langle W_{\epsilon}\right\rangle \log \max _{1 \leq j \leq s}\left|g_{j}(P)\right| .
$$


Hence by (2.2.4), (2.2.6) and (2.2.7), we have (2.2.5).

Proof of (2). Let $\lambda_{Z}$ and $\lambda_{Z}^{\prime}$ be two Weil functions for $Z$. By (3), we have $\lambda_{Z} \equiv\langle V\rangle \lambda_{Z}^{\prime}$. Hence there are a continuous function $\gamma$ on $V$ and a non-empty Zariski open subset $U \subset V-\operatorname{supp} Z$ such that

$$
\left|\lambda_{Z}(P)-\lambda_{Z}^{\prime}(P)\right| \leq \gamma(P) \quad \text { on } U
$$

(Here note that since $U$ is dense in $V-\operatorname{supp} Z$ in the usual Hausdorff topology, the above inequality also holds on $V-\operatorname{supp} Z$.) Now suppose $V$ is projective. Then the continuous function $\gamma$ is bounded, so $\lambda_{Z}=\lambda_{Z}^{\prime}+O(1)$.

Proof of (1). Take a finite affine covering $\left\{U_{\epsilon}\right\}_{\epsilon \in \Upsilon}$ of $V$, and take a system of generators $f_{1}^{\epsilon}, \ldots, f_{r_{\epsilon}}^{\epsilon} \in \Gamma\left(U_{\epsilon}, \mathcal{O}_{U_{\epsilon}}\right)$ of the ideal $\Gamma\left(U_{\epsilon}, \mathcal{I}_{Z}\right) \subset$ $\Gamma\left(U_{\epsilon}, \mathcal{O}_{U_{\epsilon}}\right)$. Set $\lambda^{\epsilon}(P)=-\log \max _{1 \leq i \leq r_{\epsilon}}\left|f_{i}^{\epsilon}(P)\right|$. Then $\lambda^{\epsilon}$ is a continuous function on $U_{\epsilon}-U_{\epsilon} \cap \operatorname{supp} Z$. Let $\left\{\rho_{\epsilon}\right\}_{\epsilon \in \Upsilon}$ be a partition of unity on $V$ consists of an open covering $\left\{U_{\epsilon}\right\}_{\epsilon \in \Upsilon}$. Set $\lambda=\sum_{\epsilon \in \Upsilon} \rho_{\epsilon} \lambda^{\epsilon}$. Then $\lambda$ is a continuous function on $V-\operatorname{supp} Z$. Note that, $\left.\lambda^{\epsilon}\right|_{U_{\epsilon} \cap U_{\kappa}}$ and $\left.\lambda^{\kappa}\right|_{U_{\epsilon} \cap U_{\kappa}}$ are both Weil functions for $\left.Z\right|_{U_{\epsilon} \cap U_{\kappa}}$. Hence by (2), there is a continuous function $\gamma_{\epsilon \kappa}$ on $U_{\epsilon} \cap U_{\kappa}$ such that $\left|\lambda^{\epsilon}-\lambda^{\kappa}\right| \leq \gamma_{\epsilon \kappa}$ on $U_{\epsilon} \cap U_{\kappa}$. We have

$$
\left|\lambda-\lambda^{\epsilon}\right| \leq \sum_{\kappa \in \Upsilon} \rho_{\kappa} \gamma_{\epsilon \kappa} \quad \text { on } U_{\epsilon}
$$

Since $\sum_{\kappa \in \Upsilon} \rho_{\kappa} \gamma_{\epsilon \kappa}$ is a continuous function on $U_{\epsilon}$, we deduce that $\lambda$ is a Weil function for $Z \subset V$.

Before going to describe various properties of $\lambda_{Z}$, we need some more definitions.

Definition 2.2.8. Let $Z, Z^{\prime}$ be closed subschemes of $V$.

(i) The intersection of $Z$ and $Z^{\prime}$, denoted $Z \cap Z^{\prime}$, is the subscheme of $V$ with the ideal sheaf $\mathcal{I}_{Z \cap Z^{\prime}}=\mathcal{I}_{Z}+\mathcal{I}_{Z^{\prime}}$.

(ii) The sum of $Z$ and $Z^{\prime}$, denoted $Z+Z^{\prime}$, is the subscheme of $V$ with the ideal sheaf $\mathcal{I}_{Z+Z^{\prime}}=\mathcal{I}_{Z} \cdot \mathcal{I}_{Z^{\prime}}$.

(iii) We say that $Z$ is contained in $Z^{\prime}$, denoted $Z \subset Z^{\prime}$, if $\mathcal{I}_{Z^{\prime}} \subset \mathcal{I}_{Z}$.

(iv) Let $\phi: V^{\prime} \rightarrow V$ be a morphism of varieties. The inverse image of $Z$, denoted $\phi^{*} Z$, is the subscheme of $V^{\prime}$ with the ideal sheaf $\mathcal{I}_{\phi^{*} Z}=\phi^{-1} \mathcal{I}_{Z} \cdot \mathcal{O}_{V}^{\prime}$ where $\phi^{-1} \mathcal{I}_{Z} \cdot \mathcal{O}_{V}^{\prime}$ is the image in $\mathcal{O}_{V}^{\prime}$ of the sheaf $\phi^{*} \mathcal{I}_{Z}$. 
Proposition 2.2.9. Let $Z, Z^{\prime}$ be closed subschemes of $V$. Then

(1) $\lambda_{Z \cap Z^{\prime}} \equiv\langle V\rangle \min \left\{\lambda_{Z}, \lambda_{Z^{\prime}}\right\}$.

(2) $\lambda_{Z+Z^{\prime}} \equiv\langle V\rangle \lambda_{Z}+\lambda_{Z^{\prime}}$.

(3) If $Z \subset Z^{\prime}$, then $\lambda_{Z} \leq\langle V\rangle \lambda_{Z^{\prime}}$.

(4) If $\operatorname{supp} Z \subset \operatorname{supp} Z^{\prime}$, then there exists a constant $c>0$ such that $\lambda_{Z} \leq\langle V\rangle c \lambda_{Z^{\prime}}$

(5) Let $U \subset V$ be a Zariski open set, and let $W$ be the complement of $U$. Suppose that $\left.\left.Z\right|_{U} \subset Z^{\prime}\right|_{U}$, then there exists a constant $c>0$ such that $\lambda_{Z} \leq\langle V\rangle \lambda_{Z^{\prime}}+c \lambda_{W}$.

(6) Let $\phi: V^{\prime} \rightarrow V$ be a morphism of varieties. Then $\lambda_{V^{\prime}, \phi^{*} Z} \equiv\left\langle V^{\prime}\right\rangle$ $\lambda_{V, Z} \circ \phi$. (Here we write $\lambda_{V, Z}$ in place of $\lambda_{Z}$ so as to better indicate the underlying variety.)

(7) Let $\phi: V^{\diamond} \rightarrow V$ be a meromorphic map. Let $U \subset V^{\diamond}$ be a Zariski open set such that $\left.\phi\right|_{U}: U \rightarrow V$ is holomorphic. Let $Z \subset V$ and $Z^{\diamond} \subset V^{\diamond}$ be closed subschemes of $V$ and $V^{\diamond}$ respectively. Suppose $\left.\phi\right|_{U} ^{*} Z=\left.Z^{\diamond}\right|_{U}$. Then there is a positive constant $c>0$ such that

$$
\lambda_{V^{\diamond}, Z^{\diamond}}-c \lambda_{V^{\diamond}, V^{\diamond}-U} \leq\left\langle V^{\diamond}\right\rangle \lambda_{V, Z} \circ \phi \leq\left\langle V^{\diamond}\right\rangle \lambda_{V^{\diamond}, Z^{\diamond}}+c \lambda_{V^{\diamond}, V^{\diamond}-U} .
$$

Proof. (1) Note that by Lemma 2.1.2 (2), it suffices to prove in the case that $V$ is affine. Let $f_{1}, \ldots, f_{r} \in \Gamma\left(V, \mathcal{O}_{V}\right)$ and $g_{1}, \ldots, g_{s} \in \Gamma\left(V, \mathcal{O}_{V}\right)$ be generators of $\mathcal{I}_{Z}$ and $\mathcal{I}_{Z^{\prime}}$, respectively. Since $f_{1}, \ldots, f_{r}, g_{1}, \ldots, g_{s}$ are generators of $\mathcal{I}_{Z \cap Z^{\prime}}$, we have

$$
\lambda_{Z \cap Z^{\prime}} \equiv\langle V\rangle-\log \max _{1 \leq i \leq r, 1 \leq j \leq s}\left\{\left|f_{i}\right|,\left|g_{j}\right|\right\} \equiv\langle V\rangle \min \left\{\lambda_{Z}, \lambda_{Z^{\prime}}\right\} .
$$

(2) We may use the same situation of the proof of (1). Since $f_{1} g_{1}, \ldots$, $f_{i} g_{j}, \ldots, f_{r} g_{s}$ are generators of $\mathcal{I}_{Z+Z^{\prime}}$, we have

$$
\lambda_{Z+Z^{\prime}} \equiv\langle V\rangle-\log \max _{1 \leq i \leq r, 1 \leq j \leq s}\left\{\left|f_{i} g_{j}\right|\right\} \equiv\langle V\rangle \lambda_{Z}+\lambda_{Z^{\prime}}
$$

(3) If $Z \subset Z^{\prime}$, then $Z=Z \cap Z^{\prime}$, so from (1), we have

$$
\lambda_{Z} \equiv\langle V\rangle \lambda_{Z \cap Z^{\prime}} \equiv\langle V\rangle \min \left\{\lambda_{Z}, \lambda_{Z^{\prime}}\right\} \leq_{\langle V\rangle} \lambda_{Z^{\prime}}
$$

(4) If $\operatorname{supp} Z \subset \operatorname{supp} Z^{\prime}$, then the Nullstellensatz implies that there is an integer $m \geq 0$ such that $\mathcal{I}_{Z} \supset \mathcal{I}_{Z^{\prime}}^{m}$. Thus $Z \subset m Z^{\prime}$, so (2) and (3) yield $\lambda_{Z} \leq\langle V\rangle m \lambda_{Z^{\prime}}$ 
(5) Using (2) and (3), it suffices to find an integer $m$ such that $Z \subset$ $Z^{\prime}+m W$; or equivalently, such that $\mathcal{I}_{Z} \supset \mathcal{I}_{Z^{\prime}} \mathcal{I}_{W}^{m}$. Since this may be checked locally, we may assume that $V=\operatorname{Spec} A$ is affine, and consider $\mathcal{I}_{Z}, \mathcal{I}_{Z^{\prime}}, \mathcal{I}_{W}$ as ideals of $A$. Writing $\mathcal{I}_{W}=\left(h_{1}, \ldots, h_{r}\right)$, we see that $U$ is the union of the open sets $U_{i}=\operatorname{Spec}\left(A_{h_{i}}\right)$. Now the given inclusion $\left.\left.Z\right|_{U} \subset Z^{\prime}\right|_{U}$ says precisely that $\mathcal{I}_{Z} A_{h_{i}} \supset \mathcal{I}_{Z^{\prime}} A_{h_{i}}$ for each $1 \leq i \leq r$. Hence we can find an integer $m$ such that $\mathcal{I}_{Z} \supset \mathcal{I}_{Z^{\prime}} h_{i}^{m}$ for all $i$; and so

$$
\mathcal{I}_{Z} \supset \mathcal{I}_{Z^{\prime}}\left(h_{1}^{m}, \ldots, h_{r}^{m}\right) \supset \mathcal{I}_{Z^{\prime}}\left(h_{1}, \ldots, h_{r}\right)^{m^{\prime}}=\mathcal{I}_{Z^{\prime}} \mathcal{I}_{W}^{m^{\prime}}
$$

(Here we may take $m^{\prime}=r m-r+1$ )

(6) We may use the same situation of the proof of (1) and (2). Let $W \subset V^{\prime}$ be a Zariski open set. Then on $W, \phi^{*} f_{1}, \ldots, \phi^{*} f_{r}$ are generators of $\mathcal{I}_{\phi^{*} Z}$. Hence, $\lambda_{V, Z} \circ \phi \equiv\langle W\rangle-\log \max _{1 \leq i \leq r}\left\{\left|\phi^{*} f_{i}\right|\right\} \equiv\langle W\rangle \lambda_{V^{\prime}, \phi^{*} Z}$. Hence by Lemma 2.1.2 (2), we have $\lambda_{V^{\prime}, \phi^{*} Z} \equiv\left\langle V^{\prime}\right\rangle \lambda_{V, Z} \circ \phi$.

(7) Let $\mu: \widetilde{V^{\diamond}} \rightarrow V^{\diamond}$ be a proper modification such that $\widetilde{\phi}=\phi \circ \mu$ : $\widetilde{V^{\diamond}} \rightarrow V$ is holomorphic and $\left.\mu\right|_{U}: \mu^{-1}(U) \rightarrow U$ is an isomorphism. By $\left.\widetilde{\phi}^{*} Z\right|_{\mu^{-1}(U)}=\left.\mu^{*} Z^{\diamond}\right|_{\mu^{-1}(U)}$ and (5), we have

$$
\lambda_{\mu^{*} Z^{\diamond}}-c^{\prime} \lambda_{\widetilde{V^{\diamond}-\mu^{-1}(U)}} \leq_{\left\langle\widetilde{\left.V^{\diamond}\right\rangle}\right.} \lambda_{\widetilde{\phi}^{*} Z} \leq_{\left\langle\widetilde{V^{\diamond}}\right\rangle} \lambda_{\mu^{*} Z^{\diamond}}+c^{\prime} \lambda_{\widetilde{V^{\diamond}}-\mu^{-1}(U)}
$$

for some positive constant $c^{\prime}>0$. By (4) and $\operatorname{supp}\left(\widetilde{V^{\diamond}}-\mu^{-1}(U)\right) \subset$ supp $\mu^{*}\left(V^{\diamond}-U\right)$, we have $\lambda_{\widetilde{V^{\diamond}}-\mu^{-1}(U)} \leq_{\left\langle\widetilde{V^{\diamond}}\right\rangle} c^{\prime \prime} \lambda_{\mu^{*}\left(V^{\diamond}-U\right)}$ for some positive constant $c^{\prime \prime}>0$. Hence by (6), we have

$$
\begin{aligned}
\lambda_{Z^{\diamond}} \circ \mu-c \lambda_{\left(V^{\diamond}-U\right)} \circ \mu & \leq_{\left\langle\widetilde{V^{\diamond}}\right\rangle} \lambda_{Z} \circ \phi \circ \mu \\
& \leq_{\left\langle\widetilde{V^{\diamond}}\right\rangle} \lambda_{Z^{\diamond}} \circ \mu+c \lambda_{\left(V^{\diamond}-U\right)} \circ \mu .
\end{aligned}
$$

for $c=c^{\prime} c^{\prime \prime}>0$.

Now since the map $\mu: \widetilde{V^{\diamond}} \rightarrow V^{\diamond}$ is proper surjective, we have the following. Let $\phi, \phi^{\prime} \in \mathcal{C}\left(V^{\diamond}\right)$. If $\phi \circ \mu \leq_{\left\langle\widetilde{V^{\diamond}}\right\rangle} \phi^{\prime} \circ \mu$, then $\phi \leq\left\langle V^{\diamond}\right\rangle \phi^{\prime}$. To show this statement, it suffices to show that for every continuous function $\alpha$ on $\widetilde{V^{\diamond}}$, there is a continuous function $\beta$ on $V^{\diamond}$ such that $\alpha \leq \beta \circ \mu$. But this can be checked easily from the fact that $\mu$ is proper. Hence (2.2.10) implies $(7)$.

A quasi-projective normal variety $V$ is said to be $\mathbb{Q}$-factorial variety if for every Weil divisor $D \subset V$, there is a positive integer $n>0$ such that $n D$ is a Cartier divisor. Hence we can consider Weil functions for all (Weil) divisors on $V$ in this case (cf. Remark $2.2 .2(1)$ ). 
LEMMA 2.2.11. Let $V$ be a quasi-projective normal and $\mathbb{Q}$-divisible variety. Let $\varphi$ be a rational function on $V$, and $(\varphi)=D_{1}-D_{2}$. Here $(\varphi)$ is the divisor defined by $\varphi$, and $D_{1}, D_{2}$ are effective (Weil) divisors. Then

$$
\log |\varphi| \equiv\langle V\rangle \lambda_{D_{2}}-\lambda_{D_{1}} .
$$

Proof. Suppose $n D_{1}$ and $n D_{2}$ are Cartier divisors. Then $\left(\varphi^{n}\right)=n D_{1}-$ $n D_{2}$, hence we can assume without loss of generality that $D_{1}$ and $D_{2}$ are Cartier divisors. By Lemma 2.1.2 (2), we can assume that $V$ is affine and $D_{1}, D_{2}$ have local equations $f_{1}, f_{2} \in \Gamma\left(V, \mathcal{O}_{V}\right)$, respectively. Then $f_{1}=\varphi f_{2} h$ (where $h \in \Gamma\left(V, \mathcal{O}_{V}\right)$ is a unit), hence we have

$$
\log |\varphi|=-\log \left|f_{2}\right|+\log \left|f_{1}\right|-\log |h| \equiv\langle V\rangle \lambda_{D_{2}}-\lambda_{D_{1}} .
$$

2.3. In this subsection, we shall prepare notations and well known results in Nevanlinna theory. Let $\pi: Y \rightarrow \mathbb{C}^{m}$ be a finite analytic covering space, i.e. $Y$ is an irreducible, normal, complex space with a finite, proper, surjective holomorphic mapping $\pi$. We denote by $k$ its sheet number and by $R(\subset Y)$ the ramification divisor of $\pi: Y \rightarrow \mathbb{C}^{m}$. Let $\left(z_{1}, \ldots, z_{m}\right)$ be the natural coordinate system in $\mathbb{C}^{m}$ and set

$$
\begin{gathered}
\|z\|=\left(\sum_{i=1}^{m}\left|z_{i}\right|^{2}\right)^{1 / 2}, \\
B(r)=\left\{z \in \mathbb{C}^{m} ;\|z\|<r\right\} \quad(r>0), \\
Y(r)=\pi^{-1}(B(r)), \\
\phi=\pi^{*} d d^{c}\|z\|^{2}=\frac{i}{2 \pi} \pi^{*} \partial \bar{\partial}\|z\|^{2}, \\
\eta=\pi^{*}\left(d^{c} \log \|z\|^{2} \wedge\left(d d^{c} \log \|z\|^{2}\right)^{m-1}\right) \\
(\eta \text { is a } 2 m-1 \text { form on } Y),
\end{gathered}
$$

where $d=\partial+\bar{\partial}$ and $d^{c}=(i / 4 \pi)(\bar{\partial}-\partial)$. For the operators $\partial, \bar{\partial}$, see for example [GH78, p. 24]. Note that

$$
\frac{1}{k} \int_{\partial Y(r)} \eta=1
$$

where $\partial Y(r)=\pi^{-1}(\partial B(r))$, and $\partial B(r)=\left\{z \in \mathbb{C}^{m} ;\|z\|=r\right\}$ for $r>0$. Let $D$ be a Weil divisor on $Y$, and define the counting function of $D$ by

$$
\mathcal{N}(D, r)=\frac{1}{k} \int_{1}^{r} \frac{d t}{t^{2 m-1}} \int_{Y(t) \cap D} \phi^{m-1} .
$$


Define the ramification counting function $N_{\pi, \operatorname{Ram}}(r)$ of the covering map $\pi: Y \rightarrow \mathbb{C}^{m}$ by

$$
N_{\pi, \operatorname{Ram}}(r)=\mathcal{N}(R, r) .
$$

Now let $f: Y \rightarrow X$ be a meromorphic map from $Y$ to a projective normal and $\mathbb{Q}$-divisible variety $X$. Let $Z \subset X$ be a closed subscheme such that $f(Y) \not \subset \operatorname{supp} Z$. Take a Weil function $\lambda_{Z}$ for $Z$. Define the proximity function of $f$ with respect to $Z$ by

$$
m_{f, Z}(r)=m(r, f, Z)=\frac{1}{k} \int_{\partial Y(r)}\left(f^{*} \lambda_{Z}\right) \eta .
$$

Since $\lambda_{Z}$ is bounded from below, $m_{f, Z}(r):(0, \infty) \rightarrow \mathbb{R}$ is also bounded from below, i.e. $0 \leq m_{f, Z}(r)+O(1)$. If we change the Weil function $\lambda_{Z}$ to $\lambda_{Z}^{\prime}$ in the above definition of $m_{f, Z}(r)$, we get a new function $m_{f, Z}^{\prime}(r)$. But the difference of these two functions is bounded, i.e. $m_{f, Z}(r)=m_{f, Z}^{\prime}(r)+$ $O(1)$ (cf. Proposition 2.2.3 (2)). We are interested in these functions only $\bmod O(1)$.

In the case that $Z$ is a Cartier divisor $D$, by Remark 2.2.2 (2), we can take the Weil function as $\lambda_{D}(P)=-\log \|\sigma(P)\|$. Hence the proximity function is

$$
m_{f, D}(r)=\frac{1}{k} \int_{\partial Y(r)} \log \frac{1}{\|\sigma \circ f\|} \eta .
$$

This is a usual definition of the proximity function (cf. [NoO90, p. 180]), and our definition is a slight generalization of this.

Next we shall define the counting function for a divisor $D$ on $X$. If $D$ is a Cartier divisor, we can define a divisor $f^{*} D$ on $Y$ and define the counting function by

$$
N_{f, D}(r)=N(r, f, D)=\mathcal{N}\left(f^{*} D, r\right)
$$

In the general case, take $n$ so that $n D$ is a Cartier divisor and put

$$
N_{f, D}(r)=N(r, f, D)=\frac{1}{n} N(r, f, n D) .
$$

Finally we define the height function $T_{f, D}(r) \bmod O(1)$ by

$$
T_{f, D}(r)=T(r, f, D)=m(r, f, D)+N(r, f, D) .
$$

Then first main Theorem in Nevanlinna Theory states: 
THEOREM 2.3.1. Let $X$ be a smooth projective variety. If two effective divisors $D$ and $D^{\prime}$ on $X$ are linearly equivalent, then

$$
T_{f, D}(r)=T_{f, D^{\prime}}(r)+O(1)
$$

The proof of this theorem can be found in [NoO90, p. 180].

By this Theorem 2.3.1, we can define the height function for a line bundle $\mathcal{O}(D)$ associated with some effective divisor $D$ to be $T_{f, \mathcal{O}(D)}(r)=$ $T_{f, D}(r)$. Since every line bundle $\mathcal{L}$ on $X$ can be written as $\mathcal{L}=\mathcal{O}(D) \otimes$ $\mathcal{O}\left(D^{\prime}\right)^{-1}$ by some effective very ample divisors $D$ and $D^{\prime}$, we shall define the height function $T_{f, \mathcal{L}}(r)$ for a line bundle $\mathcal{L}$ to be

$$
T_{f, \mathcal{L}}(r)=T_{f, \mathcal{O}(D)}(r)-T_{f, \mathcal{O}\left(D^{\prime}\right)}(r) .
$$

Now we again come back to the case where $X$ is a normal projective variety, which is possibly singular. We shall describe some properties of $m_{f, Z}(r)$ which can be checked from Proposition 2.2.9 easily.

Proposition 2.3.2. Let $X$ be a normal projective variety. Let $Z, Z^{\prime}$ be closed subschemes of $X$. Let $f: Y \rightarrow X$ be a meromorphic map such that $f(Y) \not \subset \operatorname{supp} Z \cup \operatorname{supp} Z^{\prime}$.

(1) $m_{f, Z+Z^{\prime}}(r)=m_{f, Z}(r)+m_{f, Z^{\prime}}(r)+O(1)$.

(2) Let $Z \subset Z^{\prime}$. Then, $m_{f, Z}(r) \leq m_{f, Z^{\prime}}(r)+O(1)$.

(3) Let $\operatorname{supp} Z \subset \operatorname{supp} Z^{\prime}$. Then there exists a constant $c>0$ such that

$$
m_{f, Z}(r) \leq c m_{f, Z^{\prime}}(r)+O(1)
$$

(4) Let $U \subset X$ be an Zariski open set, and let $V$ be the complement of $U$. Suppose that $\left.\left.Z\right|_{U} \subset Z^{\prime}\right|_{U}$. Then there exists a constant $c>0$ such that

$$
m_{f, Z}(r) \leq m_{f, Z^{\prime}}(r)+c m_{f, V}(r)+O(1)
$$

(5) Let $\phi: X \rightarrow X^{\prime}$ be a morphism of normal projective varieties. Let $W \subset X^{\prime}$ be a closed subscheme of $X^{\prime}$ and suppose that $f(Y) \not \subset \phi^{*} W$. Then

$$
m_{f, \phi^{*} W}(r)=m_{\phi \circ f, W}(r)+O(1) .
$$

(6) Let $\phi: X \rightarrow X^{\prime}$ be a meromorphic map. Let $U \subset X$ be a Zariski open set such that $\left.\phi\right|_{U}: U \rightarrow X^{\prime}$ is holomorphic. Let $Z \subset X$ and $W \subset X^{\prime}$ 
be closed subschemes such that $\left.\phi\right|_{U} ^{*} W=\left.Z\right|_{U}$. Suppose that $f(Y) \not \subset Z$ and $f(Y) \not \subset X-U$. Then there is a positive constant $c>0$ such that

$$
\begin{aligned}
m_{f, Z}(r)-c m_{f, X-U}(r) & \leq m_{\phi \circ f, W}(r)+O(1) \\
& \leq m_{f, Z}(r)+c m_{f, X-U}(r)+O(1) .
\end{aligned}
$$

Proof. Proposition 2.3.2 (1), (2), (3), (4), (5), (6) are obtained directly from Proposition 2.2.9 (2), (3), (4), (5), (6), (7), respectively. Here we only demonstrate Proposition 2.3.2 (1).

By Proposition 2.2.9 (2), there is a positive constant $c>0$ such that

$$
\lambda_{Z+Z^{\prime}}-c \leq \lambda_{Z}+\lambda_{Z^{\prime}} \leq \lambda_{Z+Z^{\prime}}+c
$$

on some Zariski open subset $U \subset X-\left(\operatorname{supp} Z \cup \operatorname{supp} Z^{\prime}\right)$. Since $\lambda_{Z+Z^{\prime}}$ and $\lambda_{Z}+\lambda_{Z^{\prime}}$ are continuous functions on $X-\left(\operatorname{supp} Z \cup \operatorname{supp} Z^{\prime}\right)$, and $U$ is dense in $X-\left(\operatorname{supp} Z \cup \operatorname{supp} Z^{\prime}\right)$, the estimate $(2.3 .3)$ holds on $X-(\operatorname{supp} Z \cup$ supp $\left.Z^{\prime}\right)$. We denote by $N(f)$ the set of indeterminacy of $f$. Then $N(f)$ is an analytic subset of $Y$. Consider the pull back of (2.3.3) by $f$. Then we have

$$
\lambda_{Z+Z^{\prime}} \circ f-c \leq \lambda_{Z} \circ f+\lambda_{Z^{\prime}} \circ f \leq \lambda_{Z+Z^{\prime}} \circ f+c
$$

on $Y-\left(N(f) \cup f^{*}(\operatorname{supp} Z) \cup f^{*}\left(\operatorname{supp} Z^{\prime}\right)\right)$. Hence $(2.3 .4)$ holds on $\partial Y(r)$ outside a measure zero subset with respect to $\eta$ (note that $\partial Y(r)$ has real dimension $2 \operatorname{dim} Y-1$, while $N(f) \cup f^{*}(\operatorname{supp} Z) \cup f^{*}\left(\operatorname{supp} Z^{\prime}\right)$ has real dimension $\leq 2 \operatorname{dim} Y-2)$. Taking integral $\int_{\partial Y(r)} \cdot \eta$ in (2.3.4), we obtain Proposition 2.3.2 (1).

2.4. We shall fix our notations related to a jet space. Let $V$ be a smooth algebraic variety over $\mathbb{C}$ and $x \in V$ be a point of $V$. Let $\mathbf{f}, \mathbf{g}$ : $(\mathbb{C}, 0) \rightarrow(V, x)$ be germs of holomorphic mappings from neighborhoods of the origin $0 \in \mathbb{C}$ into $V$ with $\mathbf{f}(0)=\mathbf{g}(0)=x \in V$. For a positive integer $k$, we write $\mathbf{f} \stackrel{k}{\sim} \mathbf{g}$ if $\mathbf{f}(z)$ and $\mathbf{g}(z)$ have the same Taylor expansions in $z$ up to order $k$ for some holomorphic local coordinate system around $x$. Then it is easily checked that the relation " $\underset{\sim}{\sim}$ " is independent of the choice of the holomorphic local coordinate system around $x$ and defines an equivalence relation on the set $\{\mathbf{f} \mid \mathbf{f}:(\mathbb{C}, 0) \rightarrow(V, x)\}$. Let $j_{k}(\mathbf{f})$ denote the equivalence class of $\mathbf{f}$ and set

$$
J_{k}(V)_{x}=\left\{j_{k}(\mathbf{f}) \mid \mathbf{f}:(\mathbb{C}, 0) \rightarrow(V, x)\right\} .
$$


Then $J_{k}(V)_{x}$ is naturally equipped with complex structure and isomorphic to $\mathbb{C}^{k \operatorname{dim} V}$. The $k$-th jet space $J_{k}(V)$ over $V$ is

$$
J_{k}(V)=\bigcup_{x \in V} J_{k}(V)_{x} .
$$

Then $J_{k}(V)$ naturally carries the structure of an algebraic variety. (If $\varphi \in$ $\Gamma\left(V, \mathcal{O}_{V}\right)$, then the function $d^{l} \varphi$ defined below is a holomorphic function on $J_{k}(V)$. This defines the structure of an algebraic variety on $J_{k}(V)$.) In particular, $J_{1}(V)$ is the tangent space $T V$. For convenience sake, set $J_{0}(V)=V$. For $k \geq l$ we have the natural forgetful morphism $p_{k, l}$ : $J_{k}(V) \rightarrow J_{l}(V)$.

Let $\varphi$ be a meromorphic function on $V$. For an integer $l(0 \leq l \leq k)$, the $l$-th derivative $d^{l} \varphi$ of $\varphi$ is a meromorphic function on $J_{k}(V)$ defined as follows. Let $j_{k}(\mathbf{f}) \in J_{k}(V)$ be the equivalence class of a germ $\mathbf{f}:(\mathbb{C}, 0) \rightarrow$ $(V, x)$. Then the value of the meromorphic function $d^{l} \varphi$ at the point $j_{k}(\mathbf{f}) \in$ $J_{k}(V)$ is

$$
d^{l} \varphi\left(j_{k}(\mathbf{f})\right)=\frac{d^{l}(\varphi \circ \mathbf{f})}{d z^{l}}(0) .
$$

The derivative $d^{l}$ is linear and satisfies the Leibniz rule

$$
\begin{gathered}
d^{l}(\varphi+\phi)=d^{l} \varphi+d^{l} \phi, \\
d^{l}(\varphi \phi)=\sum_{i=0}^{l}{ }_{l} \mathbf{C}_{i} d^{l-i} \varphi d^{i} \phi,
\end{gathered}
$$

where ${ }_{l} \mathbf{C}_{i}$ is binomial coefficients. If $\varphi$ is holomorphic, then $d^{l} \varphi$ is also holomorphic.

By $\bar{J}_{k}(V)$, we mean the compactification of $J_{k}(X)$ obtained by the following manner. Let $j_{k}(\mathbf{f})$ be the equivalence class of a germ $\mathbf{f}:(\mathbb{C}, 0) \rightarrow$ $(V, x)$, and $a \in \mathbb{C}^{*}=\mathbb{C}-\{0\}$ be a nonzero scalar. Then the map $j_{k}(\mathbf{f}(z)) \mapsto$ $j_{k}(\mathbf{f}(a z))$ defines the $\mathbb{C}^{*}$-action on $J_{k}(V)_{x}$. Consider the $\mathbb{C}^{*}$-action on $\mathbb{C}$ by $z \mapsto a z$ where $z \in \mathbb{C}$ and $a \in \mathbb{C}^{*}$. And consider the diagonal $\mathbb{C}^{*}$-action on $J_{k}(V)_{x} \times \mathbb{C}$. Put

$$
\bar{J}_{k}(V)_{x}=\left(J_{k}(V)_{x} \times \mathbb{C}-(\text { zero })\right) / \mathbb{C}^{*} .
$$

Then $\bar{J}_{k}(V)_{x}$ is the weighted projective space. Put

$$
\bar{J}_{k}(V)=\bigcup_{x \in V} \bar{J}_{k}(V)_{x}
$$


Then $\bar{J}_{k}(V)$ is a normal algebraic variety and has only quotient singularities, so a $\mathbb{Q}$-divisible variety. If $V$ is projective, $\bar{J}_{k}(V)$ is also projective. In particular, $\bar{T} V=\bar{J}_{1}(V)$ is smooth. We embed $J_{k}(V)_{x}$ into $\bar{J}_{k}(X)_{x}$ by sending the point $j_{k}(\mathbf{f}) \in J_{k}(V)_{x}$ to the point represented by $\left(j_{k}(\mathbf{f}), 1\right)$, which gives the open immersion $J_{k}(V) \hookrightarrow \bar{J}_{k}(V)$.

Here we give one proposition.

Proposition 2.4.3. Let $V$ be a smooth affine variety. If $\varphi_{1}, \ldots, \varphi_{d} \in$ $\Gamma\left(V, \mathcal{O}_{V}\right)(d=\operatorname{dim} V)$ form a local coordinate system around every point of $V$, then we have

$$
J_{k}(V) \simeq V \times \mathbb{C}^{k \operatorname{dim} V} \simeq V \times \operatorname{Spec} \mathbb{C}\left[d \varphi_{1}, \ldots, d \varphi_{d}, \ldots, d^{k} \varphi_{1}, \ldots, d^{k} \varphi_{d}\right]
$$

Proof. Consider the morphism

$$
\Phi: J_{k}(V) \longrightarrow V \times \operatorname{Spec} \mathbb{C}\left[d \varphi_{1}, \ldots, d \varphi_{d}, \ldots, d^{k} \varphi_{1}, \ldots, d^{k} \varphi_{d}\right]
$$

where $\Phi=\left(\operatorname{id}_{V} \circ p_{k, 0}, d \varphi_{1}, \ldots, d \varphi_{d}, \ldots, d^{k} \varphi_{1}, \ldots, d^{k} \varphi_{d}\right)$. Then $\Phi$ is an isomorphism, which proves our assertion (cf. [NoO90, Lemma 6.3.1]).

Next, for the convenience of the later use, we describe algebraic setting of a jet space. Since the fiber bundle $p=p_{k, 0}: J_{k}(V) \rightarrow V$ is locally trivial in Zariski open set of $V$ and a fiber $J_{k}(V)_{x}$ is an affine space, $p: J_{k}(V) \rightarrow$ $V$ is an affine morphism, and $J_{k}(V)=\operatorname{Spec} p_{*} \mathcal{O}_{J_{k}(V)}$. Here Spec is a spectrum of a sheaf of algebras (see for example Hartshorne [H77]). The $\mathbb{C}^{*}$-action on $J_{k}(V)_{x}$ defines the $\mathbb{C}^{*}$-action on $p_{*} \mathcal{O}_{J_{k}(V)}$ by $\tau_{a}: g\left(j_{k}(\mathbf{f}(z))\right) \mapsto$ $g\left(j_{k}(\mathbf{f}(a z))\right)$ where $g \in p_{*} \mathcal{O}_{J_{k}(V)}$. We say that $g \in p_{*} \mathcal{O}_{J_{k}(V)}$ is of weight $l$, if $\tau_{a}(g)=a^{l} g$ for every $a \in \mathbb{C}^{*}$. Let $p_{*} \mathcal{O}_{J_{k}(V), l} \subset p_{*} \mathcal{O}_{J_{k}(V)}$ be the sheaf of subabelian group of all elements of weight $l$. Then $p_{*} \mathcal{O}_{J_{k}(V), 0}=\mathcal{O}_{V}$ and $d^{l} g \in p_{*} \mathcal{O}_{J_{k}(V), l}$ for $g \in \mathcal{O}_{V}$. We have a decomposition $p_{*} \mathcal{O}_{J_{k}(V)}=$ $\bigoplus_{l \geq 0} p_{*} \mathcal{O}_{J_{k}(V), l}$ which make $p_{*} \mathcal{O}_{J_{k}(V)}$ a sheaf of graded ring. Let $\mathbb{C}\left[\eta_{\infty}\right]$ be a graded polynomial ring with a weight of $\eta_{\infty}$ to be 1 . Consider a sheaf of a graded ring $p_{*} \mathcal{O}_{J_{k}(V)} \bigotimes_{\mathcal{O}_{V}} \mathbb{C}\left[\eta_{\infty}\right]$. Then $\bar{J}_{k}(V)$ is $\operatorname{Proj}\left(p_{*} \mathcal{O}_{J_{k}(V)} \bigotimes_{\mathcal{O}_{V}}\right.$ $\left.\mathbb{C}\left[\eta_{\infty}\right]\right)$. Let $\partial J_{k}(V)$ be the $\mathbb{Q}$-Cartier divisor defined by $\eta_{\infty}$. Then $\bar{J}_{k}(V)-$ $\operatorname{supp} \partial J_{k}(V)=J_{k}(V)$. In general, let $\alpha$ be a homogeneous element of $p_{*} \mathcal{O}_{J_{k}(V)} \bigotimes_{\mathcal{O}_{V}} \mathbb{C}\left[\eta_{\infty}\right]$. Then $\alpha$ defines a $\mathbb{Q}$-Cartier divisor $(\alpha)$ and a closed subscheme $V(\alpha)$ on $\bar{J}_{k}(V)$ as follows. First, let $l$ be a weight of $\alpha$. There is a positive integer $n$ such that a sheaf (on Proj $\left(p_{*} \mathcal{O}_{J_{k}(V)} \bigotimes_{\mathcal{O}_{V}} \mathbb{C}\left[\eta_{\infty}\right]\right)$ ) associated with a sheaf (on $V)$ of twisted graded module $\left(p_{*} \mathcal{O}_{J_{k}(V)} \bigotimes_{\mathcal{O}_{V}}\right.$ 
$\left.\mathbb{C}\left[\eta_{\infty}\right]\right)(\ln )$ is a locally free sheaf of rank 1 . Then $\alpha^{n}$ defines the Cartier divisor $\left(\alpha^{n}\right)$, hence $\alpha$ defines the $\mathbb{Q}$-Cartier divisor $(\alpha)=\frac{1}{n}\left(\alpha^{n}\right)$. Next since $\alpha$ defines the homogeneous ideal of $p_{*} \mathcal{O}_{J_{k}(V)} \bigotimes_{\mathcal{O}_{V}} \mathbb{C}\left[\eta_{\infty}\right]$, hence defines the closed subscheme $V(\alpha)$. Then a straightforward computation gives the following assertion: If $n$ is a positive integer such that $n(\alpha)$ is a Cartier divisor, then we have $n(\alpha) \subset n V(\alpha)$ as closed subschemes of $\bar{J}_{k}(V)$. Hence by Proposition 2.2.9 (3), we have

$$
\lambda_{(\alpha)} \leq_{\left\langle\bar{J}_{k}(V)\right\rangle} \lambda_{V(\alpha)} .
$$

For $g \in \mathcal{O}_{V}$ and $l \leq k$, the element $d^{l} g$, regarded as a section of $p_{*} \mathcal{O}_{J_{k}(V)}$, will be written just $d^{l} g$, and when regarded as a section of $p_{*} \mathcal{O}_{J_{k}(V)} \bigotimes_{\mathcal{O}_{V}}$ $\mathbb{C}\left[\eta_{\infty}\right]$, we shall write $d^{l} g \otimes 1$. Hence $d^{l} g$ is a meromorphic function on $\bar{J}_{k}(V)$ and $d^{l} g=\left(d^{l} g \otimes 1\right) / \eta_{\infty}^{l}$.

Let $Z \subset V$ be a closed subscheme of $V$. We shall define the closed subscheme $Z^{(l)}$ of $\bar{J}_{l}(V)$ as follows. When $V$ is affine, let $\mathcal{I}=\left(g_{1}, \ldots, g_{r}\right) \subset$ $\Gamma\left(V, \mathcal{O}_{V}\right)$ be the defining ideal of the closed subscheme $Z \subset V$. Then we define the closed subscheme $Z^{(l)}$ of $\bar{J}_{l}(V)$ by the homogeneous ideal

$$
\left(g_{1}, \ldots, g_{r}, d g_{1} \otimes 1, \ldots, d g_{r} \otimes 1, \ldots, d^{l} g_{1} \otimes 1, \ldots, d^{l} g_{r} \otimes 1\right)
$$

of $p_{*} \mathcal{O}_{J_{k}(V)} \bigotimes_{\mathcal{O}_{V}} \mathbb{C}\left[\eta_{\infty}\right]$. Then by (2.4.1), (2.4.2), the above definition of $Z^{(l)}$ is well-defined. In case $V$ is general, cover $V$ with open affines $\left\{U_{i}\right\}$ and make closed subschemes $\left(\left.Z\right|_{U_{i}}\right)^{(l)} \subset \bar{J}_{l}\left(U_{i}\right)$. Then by the definition of $\left(\left.Z\right|_{U_{i}}\right)^{(l)}$, we can glue these subschemes and define the subscheme $Z^{(l)}$ of $\bar{J}_{l}(V)$.

Let $\pi: Y \rightarrow \mathbb{C}^{m}$ be a finite analytic covering space. Let $R(\subset Y)$ be the ramification divisor of $\pi$ and let $z=\left(z_{1}, \ldots, z_{m}\right)$ be the natural coordinate system in $\mathbb{C}^{m}$. We regard the vector fields $\partial / \partial z_{i}$ as meromorphic vector fields on $Y$ which are holomorphic on $Y-\operatorname{supp} R$.

Let $f: Y \rightarrow V$ be a meromorphic map to a smooth quasi-projective variety $V$. Then by considering $f_{*}\left(\partial / \partial z_{i}\right)$, we obtain the meromorphic lifting $\partial_{z_{i}}(f): Y \rightarrow \bar{T} V$ of $f$. In case $\operatorname{dim} Y=1$, by considering $f_{*}\left(\partial^{l} / \partial z^{l}\right)$, we obtain the lifting $j_{l}(f): Y \rightarrow \bar{J}_{l}(V)$ where $z=z_{1}$ and $j_{1}(f)=\partial_{z_{1}}(f)$. Note that $j_{l}(f)$ is holomorphic on $Y$ in this case. Then by the definition, for a meromorphic function $\varphi$ on $V$, we have

$$
\frac{\partial}{\partial z_{i}}(\varphi \circ f)(z)=d \varphi \circ \partial_{z_{i}}(f)(z)
$$


and in the case $\operatorname{dim} Y=1$, we have

$$
\frac{\partial^{l}}{\partial z^{l}}(\varphi \circ f)(z)=d^{l} \varphi \circ j_{l}(f)(z) .
$$

Remark 2.4.7. By some straightforward computations using a local coordinate system, we can show

$$
N\left(r, j_{l}(f), \partial J_{l}(V)\right) \leq N_{\pi, \operatorname{Ram}}(r)+\left(1-\frac{1}{l}\right) \bar{N}_{\pi, \operatorname{Ram}}(r)
$$

where $\bar{N}_{\pi, \operatorname{Ram}}(r)$ is defined by

$$
\bar{N}_{\pi, \operatorname{Ram}}(r)=\mathcal{N}(\operatorname{supp} R, r) .
$$

2.5. Next Theorem 2.5.1, called Lemma on logarithmic derivatives, is most basic in our paper. Lemma on logarithmic derivatives is first discussed by Nevanlinna [Nev39] for a meromorphic function on $\mathbb{C}$, and then by Vitter [Vi77] for a meromorphic function on $\mathbb{C}^{m}$. Following general form is obtained by Noguchi [No85].

THEOREM 2.5.1. Let $\xi$ be a meromorphic function on $Y$ considered as a meromorphic map $Y \rightarrow \mathbb{P}^{1}$. Then for each $1 \leq i \leq m$, we have

$$
\int_{\partial Y(r)} \log ^{+}\left|\frac{\frac{\partial}{\partial z_{i}} \xi}{\xi}\right| \eta \leq O\left(\log ^{+} r T(r, \xi, \infty)\right) \quad \| .
$$

Using (1) successively, we can show that the formula (1) holds if we replace $\partial / \partial z_{i}$ by $\partial^{j_{1}+\cdots+j_{m}} / \partial z_{1}^{j_{1}} \cdots \partial z_{m}^{j_{m}}$. In the case $\operatorname{dim} Y=1$, we have the following.

$$
\int_{\partial Y(r)} \log ^{+}\left|\frac{\frac{\partial^{l}}{\partial z^{l}} \xi}{\xi}\right| \eta \leq O\left(\log ^{+} r T(r, \xi, \infty)\right) \quad \| .
$$

Here the symbol $\|$ means that the inequality holds for $r \geq 0$ outside a set of finite linear measure and $\log ^{+} x=\max (0, \log x)$.

\section{$\S 3$. Formulation and proof of ANLD}

3.1. Let $X$ be a smooth projective variety and $Z \subset X$ be a closed subscheme. Let $A, B$ be sets of rational functions on $X$. We consider the following two conditions (a), (b) $Z$ on $A, B$ respectively. 
(a) For every point $P \in X$, there is a Zariski open neighborhood $U$ of $P$ and rational functions $\varphi_{1}, \ldots, \varphi_{d} \in A$ such that $\varphi_{1}, \ldots, \varphi_{d}$ are holomorphic on $U$ and form a local coordinate system around every point of $U$. Here $d=\operatorname{dim} X$.

(b) ${ }_{Z}$ For every point $P \in X$, there is a Zariski open neighborhood $U$ of $P$ and rational functions $\varphi_{1}, \ldots, \varphi_{j} \in B$ such that $\varphi_{1}, \ldots, \varphi_{j}$ are holomorphic on $U$ and $\varphi_{1}=0, \ldots, \varphi_{j}=0$ are defining equations for $Z$ on $U$. Here $j$ depends on $P$ and $U$.

Since $X$ is smooth and Noetherian, we can find finite sets $A$ and $B$ of rational functions on $X$ which satisfy conditions (a) and (b) $Z$ respectively.

For an integer $n \geq 1$, define the $n$-fold fiber product

$$
\bar{J}_{l}(X)^{n}=\underbrace{\bar{J}_{l}(X) \times_{X} \cdots \times_{X} \bar{J}_{l}(X)}_{n \text { times }}
$$

over $X$. Let $\widetilde{p}: \bar{J}_{l}(X)^{n} \rightarrow X$ be the projection, and let $q_{i}: \bar{J}_{l}(X)^{n} \rightarrow \bar{J}_{l}(X)$ be the $i$-th projection for $1 \leq i \leq n$. Put (cf. 2.2.8)

$$
Z^{(l), n}=q_{1}^{*} Z^{(l)} \cap \cdots \cap q_{n}^{*} Z^{(l)} .
$$

Then $Z^{(l), n}$ is a closed subscheme of $\bar{J}_{l}(X)^{n}$.

Proposition 3.1.1. Let $X, Z$ be as above. Suppose that $A$ and $B$ are finite sets of rational functions on $X$ satisfing the conditions (a) and $(\mathrm{b})_{Z}$ respectively. Then we have

(1) $\lambda_{\partial J_{l}(X)} \leq \sum_{\varphi \in A} \sum_{1 \leq s \leq l} \log ^{+}\left|\frac{d^{s} \varphi}{\varphi}\right|+O(1)$ on $\bar{J}_{l}(X)$,

(2) $\quad \lambda_{Z} \circ \widetilde{p} \leq \lambda_{Z^{(l), n}}+\sum_{\varphi \in B} \sum_{1 \leq i \leq n} \sum_{1 \leq s \leq l} \log ^{+}\left|\frac{d^{s} \varphi}{\varphi} \circ q_{i}\right|+O(1)$ on $\bar{J}_{l}(X)^{n}$.

Proof. Take $P \in X$. By Lemma 2.1.2 (2), it suffices to check

$$
\lambda_{\partial J_{l}(X)} \leq_{\left\langle J_{l}(U)\right\rangle} \sum_{\varphi \in A} \sum_{1 \leq s \leq l} \log ^{+}\left|\frac{d^{s} \varphi}{\varphi}\right|
$$


and

$$
\lambda_{Z} \circ \widetilde{p} \leq_{\left\langle\bar{J}_{l}(U)^{n}\right\rangle} \lambda_{Z^{(l), n}}+\sum_{\varphi \in B} \sum_{1 \leq i \leq n} \sum_{1 \leq s \leq l} \log ^{+}\left|\frac{d^{s} \varphi}{\varphi} \circ q_{i}\right|
$$

for some Zariski open neighbourhood $U$ of $P$. Let $\varphi$ be a rational function on $X$ which is holomorphic on $U$. Then on $\bar{J}_{l}(U)=p_{l, 0}^{-1}(U)$ we have

$$
|\varphi| \max \left(1,\left|\frac{d^{s} \varphi}{\varphi}\right|\right)=\max \left(|\varphi|,\left|d^{s} \varphi\right|\right)
$$

hence

$$
-\log |\varphi|=\min \left(-\log |\varphi|,-\log \left|d^{s} \varphi\right|\right)+\log \max \left(1,\left|\frac{d^{s} \varphi}{\varphi}\right|\right)
$$

for $1 \leq s \leq l$. Since $d^{s} \varphi=\left(d^{s} \varphi \otimes 1\right) / \eta_{\infty}^{s}$, we have $\left(d^{s} \varphi\right)=\left(d^{s} \varphi \otimes 1\right)-s\left(\eta_{\infty}\right)$ for the $\mathbb{Q}$-Cartier divisors. By Lemma 2.2.11, we have

$$
\lambda_{V(\varphi)} \leq_{\left\langle\bar{J}_{l}(U)\right\rangle} \min \left(\lambda_{V(\varphi)}, \lambda_{V\left(d^{s} \varphi \otimes 1\right)}-s \lambda_{\left(\eta_{\infty}\right)}\right)+\log ^{+}\left|\frac{d^{s} \varphi}{\varphi}\right|
$$

and

$$
\lambda_{V(\varphi)}+\lambda_{\left(\eta_{\infty}\right)} \leq_{\left\langle\bar{J}_{l}(U)\right\rangle} \min \left(\lambda_{V(\varphi)}+\lambda_{\left(\eta_{\infty}\right)}, \lambda_{V\left(d^{s} \varphi \otimes 1\right)}\right)+\log ^{+}\left|\frac{d^{s} \varphi}{\varphi}\right| .
$$

Hence by

$$
\begin{aligned}
& \min \left(\lambda_{V(\varphi)}+\lambda_{\left(\eta_{\infty}\right)}, \lambda_{V\left(d^{s} \varphi \otimes 1\right)}\right) \\
& \left.\quad \leq \bar{J}_{l}(U)\right\rangle \min \left(\lambda_{V(\varphi)}+\lambda_{\left(\eta_{\infty}\right)}, \lambda_{V\left(d^{s} \varphi \otimes 1\right)}+\lambda_{V(\varphi)}\right) \\
& \left.\quad \equiv \bar{J}_{l}(U)\right\rangle \\
& \min \left(\lambda_{\left(\eta_{\infty}\right)}, \lambda_{V\left(d^{s} \varphi \otimes 1\right)}\right)+\lambda_{V(\varphi)}
\end{aligned}
$$

and using (2.4.4), we have

$$
\lambda_{\left(\eta_{\infty}\right)} \leq_{\left\langle\bar{J}_{l}(U)\right\rangle} \min \left(\lambda_{V\left(\eta_{\infty}\right)}, \lambda_{V\left(d^{s} \varphi \otimes 1\right)}\right)+\log ^{+}\left|\frac{d^{s} \varphi}{\varphi}\right| .
$$

By the same argument, we have

$$
\lambda_{V(\varphi)} \leq_{\left\langle\bar{J}_{l}(U)\right\rangle} \min \left(\lambda_{V(\varphi)}, \lambda_{V\left(d^{s} \varphi \otimes 1\right)}\right)+\log ^{+}\left|\frac{d^{s} \varphi}{\varphi}\right| .
$$


Now take $U, \varphi_{1}, \ldots, \varphi_{d} \in A(d=\operatorname{dim} X)$ as condition (a). Then by Proposition 2.4.3, we have

$$
J_{l}(U)=U \times \mathbb{C}^{l d}=U \times \operatorname{Spec} \mathbb{C}\left[d \varphi_{1}, \ldots, d \varphi_{d}, \ldots, d^{l} \varphi_{1}, \ldots, d^{l} \varphi_{d}\right] .
$$

Hence we have

$$
\bar{J}_{l}(U)=U \times \operatorname{Proj} \mathbb{C}\left[d \varphi_{1} \otimes 1, \ldots, d \varphi_{d} \otimes 1, \ldots, d^{l} \varphi_{1} \otimes 1, \ldots, d^{l} \varphi_{d} \otimes 1, \eta_{\infty}\right] .
$$

Using (3.1.2) for $\varphi=\varphi_{1}, \ldots, \varphi_{d}$, we obtain the inequality

$$
\lambda_{\left(\eta_{\infty}\right)} \leq\left\langle\bar{J}_{l}(U)\right\rangle \min _{\substack{1 \leq s \leq l \\ 1 \leq t \leq d}}\left\{\lambda_{V\left(\eta_{\infty}\right)}, \lambda_{V\left(d^{s} \varphi_{t} \otimes 1\right)}\right\}+\sum_{\varphi \in A} \sum_{1 \leq s \leq l} \log ^{+}\left|\frac{d^{s} \varphi}{\varphi}\right|
$$

By Proposition $2.2 .9(1)$ and $V\left(\eta_{\infty}, d^{1} \varphi_{1} \otimes 1, \ldots, d^{l} \varphi_{d} \otimes 1\right)=\phi$, we have

$$
\min _{\substack{1 \leq s \leq l \\ 1 \leq t \leq d}}\left\{\lambda_{V\left(\eta_{\infty}\right)}, \lambda_{V\left(d^{s} \varphi_{t} \otimes 1\right)}\right\} \equiv\left\langle\bar{J}_{l}(U)\right\rangle
$$

Then inequalities (3.1.4) and (3.1.5) prove Proposition 3.1.1 (1). Note that by the definition, we have $\lambda_{\partial J_{l}(X)}=\lambda_{\left(\eta_{\infty}\right)}\left(\partial J_{l}(X)=\left(\eta_{\infty}\right)\right.$ is the definition).

Next take $U, \varphi_{1}, \ldots, \varphi_{j} \in B$ satisfying the condition (b) $Z$. Using (3.1.3) for $\varphi=\varphi_{1}, \ldots, \varphi_{j}$ and taking compositions with $q_{i}$ 's, we have

$$
\begin{aligned}
& \min _{1 \leq t \leq j}\left\{\lambda_{V\left(\varphi_{t}\right)}\right\} \leq_{\left\langle\bar{J}_{l}(U)^{n}\right\rangle} \min _{1 \leq i \leq n} \min _{1 \leq s \leq l}\left\{\lambda_{V\left(\varphi_{t}\right)}, \lambda_{V\left(d^{s} \varphi_{t} \otimes 1\right)} \circ q_{i}\right\} \\
& 1 \leq t \leq j \\
& +\sum_{\varphi \in B} \sum_{1 \leq i \leq n} \sum_{1 \leq s \leq l} \log ^{+}\left|\frac{d^{s} \varphi}{\varphi} \circ q_{i}\right| \text {. }
\end{aligned}
$$

By condition $(\mathrm{b})_{Z}$, we have $Z=V\left(\varphi_{1}, \ldots, \varphi_{j}\right)$ and by definition of $Z^{(l)}$ and $Z^{(l), n}$, we have $Z^{(l)}=V\left(\varphi_{1}, \ldots, d^{l} \varphi_{j} \otimes 1\right)$ and $Z^{(l), n}=q_{1}^{*} Z^{(l)} \cap \cdots \cap q_{n}^{*} Z^{(l)}$. Hence Proposition 2.2.9 (1) and (3.1.6) prove Proposition 3.1.1 (2).

3.2. Let $\pi: Y \rightarrow \mathbb{C}^{m}$ be a finite analytic covering space, and let $f: Y \rightarrow X$ be a meromorphic map. Set $\partial(f)=\partial_{z_{1}}(f) \times \cdots \times \partial_{z_{m}}(f)$. Then $\partial(f)$ is a lifting of $f$;

$$
\partial(f): Y \rightarrow \bar{T} X^{m}=\underbrace{\bar{T} X \times_{X} \cdots \times_{X} \bar{T} X}_{m \text { times }} .
$$


In the rest of this paper, $S(r, f)$ denotes any small term satisfying

$$
S(r, f) \leq O\left(\log ^{+} r T(r, f, E)\right) \quad \| .
$$

Here $E$ is an ample line bundle on $X$. If we take another ample line bundle $E^{\prime}$ on $X$, we have $O(T(r, f, E))=O\left(T\left(r, f, E^{\prime}\right)\right)$. Hence our notation $S(r, f)$ is independent of the choice of an ample line bundle on $X$.

THEOREM 3.2.1. (ANLD) Let $X$ be a smooth projective variety, and let $Z \subset X$ be a closed subscheme. Let $\pi: Y \rightarrow \mathbb{C}^{m}$ be a finite analytic covering space. Let $f: Y \rightarrow X$ be a meromorphic map. Suppose $f(Y) \not \subset \operatorname{supp} Z$. Then we have

$$
\begin{aligned}
& m(r, f, Z) \leq m\left(r, \partial(f), Z^{(1), m}\right)+S(r, f) . \\
& m\left(r, \partial(f), q_{1}^{*}(\partial T X)+\cdots+q_{m}^{*}(\partial T X)\right) \leq S(r, f) .
\end{aligned}
$$

Moreover if $\operatorname{dim} Y=1$, we have

$$
\begin{aligned}
& m(r, f, Z) \leq m\left(r, j_{l}(f), Z^{(l)}\right)+S(r, f) \\
& m\left(r, j_{l}(f), \partial J_{l}(X)\right) \leq S(r, f)
\end{aligned}
$$

Proof. First, note that since $X$ is smooth and projective, we can take finite sets $A$ and $B$ of rational functions on $X$ which are finite and satisfy the conditions (a) and $(\mathrm{b})_{Z}$ respectively. Moreover we can take $A, B$ such that

$$
f(Y) \not \subset \bigcup_{\varphi \in A \cup B}(\varphi)_{0} \cup(\varphi)_{\infty} .
$$

This means that $\varphi \circ f$ is a meromorphic function on $Y$ with $\varphi \circ f \neq 0, \infty$ for every $\varphi \in A \cup B$. By pulling back the inequality of Proposition 3.1.1 (2) by meromorphic map $\partial(f)$ (in case $l=1, n=m$ ) and using (2.4.5), we obtain

$$
\lambda_{Z} \circ f \leq \lambda_{Z^{(1), m}} \circ \partial(f)+\sum_{\varphi \in B} \sum_{1 \leq i \leq m} \log ^{+}\left|\frac{\frac{\partial}{\partial z_{i}}(\varphi \circ f)}{(\varphi \circ f)}\right|+O(1) .
$$

Then taking the integral $\int_{\partial Y(r)} \cdot \eta$ and using Theorem 2.5.1 (1), we prove Theorem 3.2.1 (1).

Next to prove (2), since we have

$$
m\left(r, \partial(f), q_{1}^{*}(\partial T X)+\cdots+q_{m}^{*}(\partial T X)\right)=\sum_{1 \leq i \leq m} m\left(r, \partial_{z_{i}}(f), \partial T X\right),
$$


it suffices to check

$$
m\left(r, \partial_{z_{i}}(f), \partial T X\right) \leq S(r, f) \quad \text { for } 1 \leq i \leq m
$$

This can be checked from Proposition 3.1.1 (1), (2.4.5) and Theorem 2.5.1 (1).

For Theorem 3.2.1 (3), use Proposition 3.1.1 (2) for $n=1$ and (2.4.6), Theorem 2.5.1 (2).

For Theorem 3.2.1 (4), use Proposition 3.1.1 (1), (2.4.6) and Theorem 2.5.1 (2).

Above Theorem 3.2.1 (3) was first observed by Kobayashi [Kob96a], [Kob96b] in somewhat weak forms (with some non-degeneracy condition on $f$ and in a weak estimate for the error term). In case that $X$ is an Abelian variety, another proof for Theorem 3.2.1 (3), (4) can be found in [Kob00].

Remark 3.2.3. By Theorem 3.2.1 (4) and (2.4.8), we have

$$
T\left(r, j_{l}(f), \partial J_{l}(X)\right) \leq N_{\pi, \operatorname{Ram}}(r)+\left(1-\frac{1}{l}\right) \bar{N}_{\pi, \operatorname{Ram}}(r)+S(r, f)
$$

for the estimate of the height function.

Remark 3.2.5. For the case $X=\mathbb{P}^{1}$ and a meromorphic map $f=\xi$ : $Y \rightarrow \mathbb{P}^{1}$, above Theorem 3.2.1 (1), (2) give usual Nevanlinna's lemma on logarithmic derivative Theorem 2.5.1 (1).

First, in general we have

$$
m\left(r, \partial(f), Z^{(1), m}\right) \leq m\left(r, \partial_{z_{i}}(f), Z^{(1)}\right)+O(1) \quad \text { for } 1 \leq i \leq m .
$$

Hence by Theorem 3.2.1 (1), we have

$$
m(r, f, Z) \leq m\left(r, \partial_{z_{i}}(f), Z^{(1)}\right)+S(r, f) \quad \text { for } 1 \leq i \leq m .
$$

By Theorem 3.2.1 (2) and its proof (cf. (3.2.2)), we have

$$
m\left(r, \partial_{z_{i}}(f), \partial T X\right) \leq S(r, f) \text { for } 1 \leq i \leq m
$$

Set $X=\mathbb{P}^{1}=\mathbb{C} \cup\{\infty\}$ and let $t$ be the coordinate on $\mathbb{P}^{1}-\{\infty\}$. Let $D_{0}$ and $D_{\infty}$ be divisors of $\mathbb{P}^{1}$ determined by $t=0$ and $t=\infty$ respectively. Let $p: \bar{T} \mathbb{P}^{1} \rightarrow \mathbb{P}^{1}$ be the projection. Let $F_{0}$ and $F_{\infty}$ be the divisors 
on $\bar{T} \mathrm{P}^{1}$ associated to the section at zero and infinity respectively. (Hence $F_{\infty}=\partial T \mathbb{P}^{1}$.) Consider the rational function $d t / t$ on $\bar{T} \mathbb{P}^{1}$. Then we have

$$
\left(\frac{d t}{t}\right)=F_{0}-p^{*} D_{0}-p^{*} D_{\infty}-F_{\infty}
$$

Hence by Lemma 2.2.11, we have

$$
\log \left|\frac{d t}{t}\right|=\lambda_{p^{*} D_{0}+p^{*} D_{\infty}+F_{\infty}}-\lambda_{F_{0}}+O(1) .
$$

Hence we have

$$
\begin{aligned}
& \log ^{+}\left|\frac{d t}{t}\right|=\max \left\{0, \log \left|\frac{d t}{t}\right|\right\}=\max \left\{0, \lambda_{p^{*} D_{0}+p^{*} D_{\infty}+F_{\infty}}-\lambda_{F_{0}}\right\}+O(1) \\
& =\lambda_{p^{*} D_{0}+p^{*} D_{\infty}+F_{\infty}}-\min \left\{\lambda_{p^{*} D_{0}+p^{*} D_{\infty}+F_{\infty}}, \lambda_{F_{0}}\right\}+O(1) .
\end{aligned}
$$

By Proposition 2.2.9 (1), we have

$$
\log ^{+}\left|\frac{d t}{t}\right|=\lambda_{p^{*} D_{0}+p^{*} D_{\infty}+F_{\infty}}-\lambda_{\left(p^{*} D_{0}+p^{*} D_{\infty}+F_{\infty}\right) \cap F_{0}}+O(1) .
$$

Since we have

$$
\left(p^{*} D_{0}+p^{*} D_{\infty}+F_{\infty}\right) \cap F_{0}=D_{0}^{(1)}+D_{\infty}^{(1)},
$$

by pulling back (3.2.8) by $\partial_{i}(\xi): Y \rightarrow \bar{T} \mathbb{P}^{1}$ and taking the integral $\int_{\partial Y(r)} \cdot \eta$, we have

$$
\begin{aligned}
\int_{\partial Y(r)} \log ^{+}\left|\frac{\frac{\partial}{\partial z_{i}} \xi}{\xi}\right| \eta=( & \\
& \left.\left(r, \xi, D_{0}\right)-m\left(r, \partial_{z_{i}}(\xi), D_{0}^{(1)}\right)\right) \\
& +\left(m\left(r, \xi, D_{\infty}\right)-m\left(r, \partial_{z_{i}}(\xi), D_{\infty}^{(1)}\right)\right) \\
& +m\left(r, \partial_{z_{i}}(\xi), \partial T \mathbb{P}^{1}\right) .
\end{aligned}
$$

From (3.2.6), (3.2.7) and (3.2.9), we deduce Theorem 2.5.1 (1).

\section{§4. Applications to the Second Main Theorem}

In this section, we shall discuss applications of our ANLD to the second main theorem in Nevanlinna theory in several complex variables. In Subsection 4.1, we shall discuss equidimensional value distribution theory. After this subsection, we shall consider the case of holomorphic curves, which is the most basic case in non-equidimensional value distribution theory. After giving some general technical result (Proposition 4.2.1) in Subsection 4.2, we shall discuss the second main theorem for Abelian varieties and for the projective space with hyperplanes in Subsections 4.3 and 4.4 respectively. 
4.1. For a line bundle $u_{L}: L \rightarrow X$ on $X$, we shall denote its dual line bundle by $u_{L^{\vee}}: L^{\vee} \rightarrow X$. Let $\bar{L}=\mathbb{P}\left(\mathcal{O}_{X} \oplus L\right)$ be the compactification of $L$, and let $u_{\bar{L}}: \bar{L} \rightarrow X$ be the projection. Let $F_{0}^{[L]}$ (resp. $F_{\infty}^{[L]}$ ) be the divisor on $\bar{L}$ associated to the section at zero (resp. infinity). Then we have

$$
u_{\bar{L}}^{*} L^{\vee}=\mathcal{O}_{\bar{L}}\left(F_{\infty}^{[L]}-F_{0}^{[L]}\right) .
$$

Let $K_{X}$ be the canonical line bundle on $X$. Let $\tau_{\wedge}$ be the holomorphic map

$$
\tau_{\wedge}: T X^{d}=\underbrace{T X \times_{X} \cdots \times_{X} T X}_{d \text { times }} \longrightarrow K_{X}^{\vee} \quad(d=\operatorname{dim} X)
$$

sending $\left(v_{1}, \ldots, v_{d}\right)$ to $v_{1} \wedge \cdots \wedge v_{d}$. Let $\bar{\tau}_{\wedge}$ be the rational map

$$
\bar{\tau}_{\wedge}: \bar{T} X^{d}=\underbrace{\bar{T} X \times_{X} \cdots \times_{X} \bar{T} X}_{d \text { times }} \rightarrow \overline{K_{X}^{\vee}}
$$

which is the extension of $\tau_{\wedge}$.

LEMmA 4.1.2. Let $D \subset X$ be a simple normal crossing divisor and write $D=\sum_{j} D_{j}$ as a sum of irreducible components. Then we have

$$
\left.\left.\sum_{j} D_{j}^{(1), d}\right|_{T X^{d}} \subset \bar{\tau}_{\wedge}^{*} F_{0}^{\left[K_{X}^{\vee}\right]}\right|_{T X^{d}}=\tau_{\wedge}^{*} F_{0}^{\left[K_{X}^{\vee}\right]} .
$$

Proof. For every point $P \in X$, there is an open neighborhood $U \subset X$ $(P \in U)$, and local functions $h_{1}, \ldots, h_{d} \in \Gamma\left(U, \mathcal{O}_{U}\right)$ such that

(1) $h_{1}, \ldots, h_{d}$ form a local coordinate system around every point on $U$.

(2) $h_{1} \cdots h_{t}=0(0 \leq t \leq d)$ is a local equation for $\left.D\right|_{U}$.

Then $T U=U \times \mathbb{C}^{d}$ and $d h_{1}, \ldots, d h_{d}$ form the coordinate system of $\mathbb{C}^{d}$ (cf. Proposition 2.4.3). Hence, $T U^{d}=U \times \mathbb{C}^{d^{2}}$ and $\left(d h_{j}^{i}\right)_{1 \leq i, j \leq d}$ forms the coordinate system of $\mathbb{C}^{d^{2}}$, where $d h_{j}^{i}=q_{i}^{*} d h_{j}$ (here $q_{i}: T U^{d} \rightarrow T U$ is the $i$-th projection). Put

$$
\Theta=\left|\begin{array}{ccc}
d h_{1}^{1} & \cdots & d h_{1}^{d} \\
\vdots & \ddots & \vdots \\
d h_{d}^{1} & \cdots & d h_{d}^{d}
\end{array}\right| .
$$

Then $\Theta=0$ is the local equation for $\tau_{\wedge}^{*} F_{0}^{\left[K_{X}^{\vee}\right]}$ on $T U^{d}$. Note that we have

$$
\Gamma\left(T U^{d}, \mathcal{I}_{\sum_{j} D_{j}^{(1), d}}\right)=\left(h_{1}, d h_{1}^{1}, \ldots, d h_{1}^{d}\right) \cdots\left(h_{t}, d h_{t}^{1}, \ldots, d h_{t}^{d}\right) .
$$


Hence to check Lemma 4.1.2, it suffices to show

$$
\Theta \in\left(h_{1}, d h_{1}^{1}, \ldots, d h_{1}^{d}\right) \cdots\left(h_{t}, d h_{t}^{1}, \ldots, d h_{t}^{d}\right)
$$

but this can be checked by straightforward computations.

Next theorem was obtained by Carlson-Griffiths [CG72], Griffiths-King [GK73], Shiffman [Sh75] and Noguchi [No76]. Here, we shall discuss this in the context of our method.

ThEOREM 4.1.3. Let $\pi: Y \rightarrow \mathbb{C}^{d}$ be a finite analytic covering space, and let $X$ be a smooth projective variety. Let $f: Y \rightarrow X$ be a meromorphic map and let $D \subset X$ be a divisor with simple normal crossings. Suppose $\operatorname{dim} Y=\operatorname{dim} X(=d)$ and $f$ is non-degenerate in the sense that the Jacobian of $f$ does not identically vanish. Then we have

$$
m(r, f, D)+N_{\operatorname{Ram} f}(r)+T\left(r, f, K_{X}\right) \leq N_{\operatorname{Ram}, \pi}(r)+S(r, f) .
$$

Proof. Write $D=\sum_{j=1}^{s} D_{j}$ as a sum of irreducible components. Then by Theorem 3.2.1 (1), we have

$$
m(r, f, D)=\sum_{j=1}^{s} m\left(r, f, D_{j}\right) \leq \sum_{j=1}^{s} m\left(r, \partial(f), D_{j}^{(1), d}\right)+S(r, f)
$$

By Lemma 4.1.2 and Proposition 2.3.2 (6), there is a positive constant $c>0$ such that

$$
\begin{aligned}
& \sum_{j=1}^{s} m\left(r, \partial(f), D_{j}^{(1), d}\right) \\
& \quad \leq m\left(r, \bar{\tau}_{\wedge} \circ \partial(f), F_{0}^{\left[K_{X}^{\vee}\right]}\right)+c \cdot m\left(r, \partial(f), \bar{T} X^{d}-T X^{d}\right) .
\end{aligned}
$$

Hence by Theorem 3.2.1 (2), we have

$$
m(r, f, D) \leq m\left(r, \bar{\tau}_{\wedge} \circ \partial(f), F_{0}^{\left[K_{X}^{\vee}\right]}\right)+S(r, f)
$$

Since

$$
m\left(r, \bar{\tau}_{\wedge} \circ \partial(f), F_{0}^{\left[K_{X}^{\vee}\right]}\right)=T\left(r, \bar{\tau}_{\wedge} \circ \partial(f), F_{0}^{\left[K_{X}^{\vee}\right]}\right)-N_{\operatorname{Ram} f}(r)+O(1)
$$


(note that $N_{\operatorname{Ram} f}(r)=N\left(r, \bar{\tau}_{\wedge} \circ \partial(f), F_{0}^{\left[K_{X}^{\vee}\right]}\right)$ by definition) and by (4.1.1) and Nevanlinna's first main theorem (Theorem 2.3.1), we have

$$
\begin{aligned}
& m(r, f, D)+N_{\operatorname{Ram} f}(r)+T\left(r, f, K_{X}\right) \\
& \quad \leq T\left(r, \bar{\tau}_{\wedge} \circ \partial(f), F_{\infty}^{\left[K_{X}^{\vee}\right]}\right)+S(r, f) .
\end{aligned}
$$

Here, note that by our non-degeneracy condition on $f$, we have $\bar{\tau} \wedge \circ \partial(f)(Y)$ $\not \subset F_{0}^{\left[K_{X}^{\vee}\right]}$. Again, using Proposition 2.3.2 (6) to the case $U=T X^{d}, Z=\phi$, $W=F_{\infty}^{\left[K_{X}^{\vee}\right]}$, we have

$$
m\left(r, \bar{\tau}_{\wedge} \circ \partial(f), F_{\infty}^{\left[K_{X}^{\vee}\right]}\right) \leq S(r, f) .
$$

Let $R$ be the ramification divisor of $\pi: Y \rightarrow \mathbb{C}^{d}$. We shall show

$$
\left(\bar{\tau}_{\wedge} \circ \partial(f)\right)^{*} F_{\infty}^{\left[K_{X}^{\vee}\right]} \subset R .
$$

We write $S(Y)$ for the set of singularities of the complex space $Y$, and $N(f)$ for the set of indeterminacy of $f$. Then $\operatorname{since} \operatorname{codim}(S(Y) \cup N(f)) \geq 2$, to show (4.1.6), it suffices to prove it on $Y-S(Y)-N(f)$. Take a point $P \in Y-S(Y)-N(f)$, and take an open neighborhood $W_{X} \ni f(P)$ and $W_{Y} \ni P$ such that $W_{Y} \subset Y-S(Y)-N(f), f\left(W_{Y}\right) \subset W_{X}$. Moreover we assume that $W_{X}, W_{Y}$ are biholomorphic to the open sets of $\mathbb{C}^{d}$. Set $x=\left(x_{1}, \ldots, x_{d}\right)$ and $y=\left(y_{1}, \ldots, y_{d}\right)$ be the coordinate systems of $W_{X}$ and $W_{Y}$ respectively. Write $f$ in these coordinate systems as

$$
x_{1}=f_{1}\left(y_{1}, \ldots, y_{d}\right), \ldots, x_{d}=f_{d}\left(y_{1}, \ldots, y_{d}\right) .
$$

Set Jacobian matrix of $f(y)$ as $\theta(y)$;

$$
\theta(y)=\left|\begin{array}{ccc}
\frac{\partial f_{1}}{\partial y_{1}}(y) & \cdots & \frac{\partial f_{1}}{\partial y_{d}}(y) \\
\vdots & \ddots & \vdots \\
\frac{\partial f_{d}}{\partial y_{1}}(y) & \cdots & \frac{\partial f_{d}}{\partial y_{d}}(y)
\end{array}\right|
$$

Let $z=\left(z_{1}, \ldots, z_{d}\right)$ be the standard coordinate system of $\mathbb{C}^{d}(\stackrel{\pi}{\leftarrow} Y)$, and define the holomorphic function $r(y)$ on $W_{Y}$ by

$$
\pi^{*}\left(d z_{1}\right) \wedge \cdots \wedge \pi^{*}\left(d z_{d}\right)=r(y) d y_{1} \wedge \cdots \wedge d y_{d}
$$

Then $r(y)=0$ is the local equation for $R$ on $W_{Y}$. Since the restriction of $\bar{\tau}_{\wedge} \circ \partial(f)$ to $W_{Y}$ is

$$
\left.\bar{\tau}_{\wedge} \circ \partial(f)\right|_{W_{Y}}(y)=\left(f(y), \frac{\theta(y)}{r(y)}\right) \in W_{X} \times \mathbb{P}^{1}=\overline{K_{W_{X}}^{\vee}},
$$


we have (4.1.6) on $W_{Y}$, hence also on $Y-S(Y)-N(f)$ and $Y$.

Now consider the integral $\mathcal{N}(\cdot, r)$ of (4.1.6). Then we obtain the inequality of the counting functions

$$
N\left(r, \bar{\tau}_{\wedge} \circ \partial(f), F_{\infty}^{\left[K_{X}^{\vee}\right]}\right) \leq N_{\operatorname{Ram}, \pi}(r)
$$

Hence (4.1.4), (4.1.5) and (4.1.7) imply Theorem 4.1.3.

4.2. In the rest of this paper, we shall mainly consider the case $Y=\mathbb{C}$, which is the most basic and simple case in non-equidimensional value distribution theory. When the following assumption of Proposition 4.2.1 is satisfied for $L=K_{X}$, our situation becomes similar to the case of equidimensional value distribution theory. Here we consider the higher jet space $J_{N}(X)$ instead of $T X^{d}$.

Proposition 4.2.1. Let $X$ be a smooth projective variety, and let $D=$ $D_{1}+\cdots+D_{s} \subset X$ be a divisor. Suppose there is a positive integer $N>0$, a line bundle $L \stackrel{u_{L}}{\longrightarrow} X$, and a morphism of fiber bundles $\psi: J_{N}(X) \rightarrow L^{\vee}$ such that $\left.D_{1}^{(N)}\right|_{J_{N}(X)}+\cdots+\left.D_{s}^{(N)}\right|_{J_{N}(X)} \subset \psi^{*} F_{0}^{\left[L^{\vee}\right]}$. Then for a holomorphic curve $f: \mathbb{C} \rightarrow X$ with a non-degeneracy condition $j_{N}(f)(\mathbb{C}) \not \subset \psi^{*} F_{0}^{\left[L^{\vee}\right]}$, we have

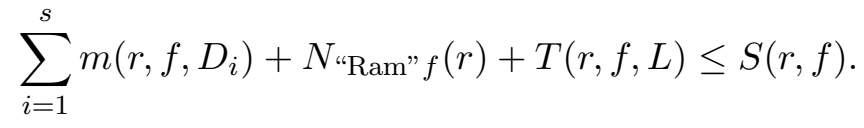

Here we define the term $N$ "Ram" $f(r)$ by $N$ "Ram" $f(r)=N\left(r, \psi \circ j_{N}(f), F_{0}^{\left[L^{\vee}\right]}\right)$.

Proof. By Theorem 3.2.1 (3), we have

$$
\sum_{i=1}^{s} m\left(r, f, D_{i}\right) \leq \sum_{i=1}^{s} m\left(r, j_{N}(f), D_{i}^{(N)}\right)+S(r, f) .
$$

By our assumption $\left.D_{1}^{(N)}\right|_{J_{N}(X)}+\cdots+\left.D_{s}^{(N)}\right|_{J_{N}(X)} \subset \psi^{*} F_{0}^{\left[L^{\vee}\right]}$, and Proposition 2.3.2 (6), there is a positive constant $c>0$ such that

$$
\begin{aligned}
& \sum_{i=1}^{s} m\left(r, j_{N}(f), D_{i}^{(N)}\right) \\
& \quad \leq m\left(r, \psi \circ j_{N}(f), F_{0}^{\left[L^{\vee}\right]}\right)+c \cdot m\left(r, j_{N}(f), \partial J_{N}(X)\right)
\end{aligned}
$$


(Here we consider the holomorphic curve $\psi \circ j_{N}(f): \mathbb{C} \rightarrow \overline{L^{\vee}}$.) Hence by Theorem 3.2.1 (4), we have

$$
\sum_{i=1}^{s} m\left(r, f, D_{i}\right) \leq m\left(r, \psi \circ j_{N}(f), F_{0}^{\left[L^{\vee}\right]}\right)+S(r, f) .
$$

By $m\left(r, \psi \circ j_{N}(f), F_{0}^{\left[L^{\vee}\right]}\right)+N$ "Ram" $f(r)=T\left(r, \psi \circ j_{N}(f), F_{0}^{\left[L^{\vee}\right]}\right),(4.1 .1)$ and Theorem 2.3.1, we have

$$
\begin{aligned}
m(r, & \left.\psi \circ j_{N}(f), F_{0}^{\left[L^{\vee}\right]}\right)+N \text { "Ram" } f(r) \\
& \leq-T(r, f, L)+T\left(r, \psi \circ j_{N}(f), F_{\infty}^{\left[L^{\vee}\right]}\right) \\
& =-T(r, f, L)+m\left(r, \psi \circ j_{N}(f), F_{\infty}^{\left[L^{\vee}\right]}\right) .
\end{aligned}
$$

Again by Proposition 2.3.2 (6) and Theorem 3.2.1 (4), we have

$$
m\left(r, \psi \circ j_{N}(f), F_{\infty}^{\left[L^{\vee}\right]}\right) \leq S(r, f) .
$$

Hence we have

$$
\sum_{i=1}^{s} m\left(r, f, D_{i}\right)+N \text { "Ram" } f(r)+T(r, f, L) \leq S(r, f) .
$$

The following corollary of Proposition 4.2.1 is the most basic technical tool for hyperbolicity problems such as Bloch's theorem ([B26]). This corollary is established by Y. T. Siu and S. K. Yeung [SiY97] using a negative curvature method. Though we don't use it in this paper any more, but it may be helpful to see how we can apply ANLD to get it. For the application of this corollary, see for example [GG80].

Corollary 4.2.3. Let $X$ be as above. Let $D \subset X$ be an ample divisor. Suppose there is a positive integer $k$ and a $k$-jet differential $\omega$ on $X$ (i.e. $\left.\omega \in \Gamma\left(X, p_{k, 0 *} \mathcal{O}_{J_{k}(X)}\right)\right)$ such that $\omega$ vanishes on $D$ but is not identically zero on $X$. Then for any holomorphic curve $f: \mathbb{C} \rightarrow X$, the pullback $f^{*} \omega$ is identically zero on $\mathbb{C}$.

Proof. Let $L_{D}$ be the line bundle over $X$ associated to $D$. Since our jet differential $\omega$ defines the holomorphic function on $J_{k}(X)$, it defines the morphism of fiber bundles $J_{k}(X) \rightarrow X \times \mathbb{C}$. Since $\omega$ vanishes on $D$, this 
morphism factor the subbundle $L_{D}^{\vee} \rightarrow X \times \mathbb{C}$. Hence we have the morphism of fiber bundles

$$
\psi: J_{k}(X) \longrightarrow L_{D}^{\vee}
$$

over $X$.

Now we shall prove our corollary by assuming that there is a holomorphic curve $f: \mathbb{C} \rightarrow X$ such that $f^{*} \omega$ is not identically zero on $\mathbb{C}$, and derive a contradiction. Assume that there is a holomorphic curve $f: \mathbb{C} \rightarrow X$ such that $f^{*} \omega$ is not identically zero on $\mathbb{C}$. This means $j_{k}(f)(\mathbb{C}) \not \subset \psi^{*} F_{0}^{\left[L_{D}^{\vee}\right]}$. Hence by Proposition 4.2.1, we have

$$
T\left(r, f, L_{D}\right) \leq S(r, f) \leq O\left(\log ^{+} r T\left(r, f, L_{D}\right)\right) \quad \|
$$

But this implies that $f$ is rational and factors some morphism $\phi: \mathbb{P}^{1} \rightarrow X$. By restricting $\psi$ on $\mathbb{P}^{1}$ by $\phi$, we obtain the morphism of fiber bundles

$$
\widetilde{\psi}: J_{k}\left(\mathbb{P}^{1}\right) \longrightarrow M^{\vee}
$$

over $\mathbb{P}^{1}$. Here $M$ is the line bundle over $\mathbb{P}^{1}$ obtained by $\phi^{*} L_{D}$. Then by $\operatorname{Pic}\left(\mathbb{P}^{1}\right) \simeq \mathbb{Z}$, we see that $M$ is ample. Note that $\widetilde{\psi}$ is not identically zero because of $j_{k}(f)(\mathbb{C}) \not \subset \psi^{*} F_{0}^{\left[L_{D}^{\vee}\right]}$. Now take a holomorphic curve $g$ : $\mathbb{C} \rightarrow \mathbb{P}^{1}$ such that $j_{k}(g)(\mathbb{C}) \not \subset \widetilde{\psi}^{*} F_{0}^{\left[M^{\vee}\right]}$ and $T(r, g, M) \geq r^{2}$. Then by Proposition 4.2.1, we have

$$
T(r, g, M) \leq S(r, g) \leq O\left(\log ^{+} r T(r, g, M)\right) \quad \|
$$

But this is a contradiction. Hence for any holomorphic curve $f: \mathbb{C} \rightarrow X$, $f^{*} \omega$ is identically zero on $\mathbb{C}$.

Remark 4.2.4. In Proposition 4.2.1, if we consider a holomorphic curve $f: Y \rightarrow X$, where $\pi: Y \rightarrow \mathbb{C}$ is a finite analytic covering space, a modification of (4.2.2) gives

$$
\sum_{i=1}^{s} m\left(r, f, D_{i}\right)+N \text { "Ram" } f(r)+T(r, f, L) \leq O\left(N_{\pi, \operatorname{Ram}}(r)\right)+S(r, f)
$$

under the non-degeneracy condition $j_{N}(f)(Y) \not \subset \psi^{*} F_{0}^{\left[L^{\vee}\right]}$. 
4.3. We shall explain how to use our Proposition 4.2.1 to obtain the second main theorem for holomorphic curves into Abelian varieties established by [Kob00] and [NoWY02].

TheOrem 4.3.1. Let $A$ be an Abelian variety, and let $D$ be an effective divisor on $A$. Let $f: \mathbb{C} \rightarrow A$ be a holomorphic curve such that $f(\mathbb{C}) \not \subset$ $\operatorname{supp} D$. Then we have

$$
m(r, f, D) \leq S(r, f)
$$

Proof. First, we shall reduce the proof to the case $f$ is non-degenerate, i.e. the Zariski closure of $f(\mathbb{C})$ is equal to $A$. Let $B$ be the Zariski closure of $f(\mathbb{C})$. Then by Bloch-Ochiai's theorem, $B$ is a translate of an Abelian subvariety of $A$. From the condition $f(\mathbb{C}) \not \subset D$, we have $m(r, f, D)=$ $m(r, f, D \cap B)$ by Proposition 2.3.2 (5). Thus it suffices to prove Theorem for the case $A$ is $B$ and $D$ is $B \cap D$, but this is the case $f$ is non-degenerate.

Next we show that there is a positive integer $k>0$ and a morphism of fiber bundles $\psi: J_{k}(A) \rightarrow K_{A}^{\vee}$ such that $\left.D^{(k)}\right|_{J_{k}(A)} \subset \psi^{*} F_{0}^{\left[K_{A}^{\vee}\right]}$ and $j_{k}(f)(\mathbb{C}) \not \subset \psi^{*} F_{0}^{\left[K_{A}^{\vee}\right]}$. This part of the argument is motivated by Kobayashi [Kob91a]. Note that we have canonically $J_{k}(A) \simeq A \times \mathbb{C}^{k \operatorname{dim} A}$. Let $r_{k}$ be the projection $J_{k}(A) \stackrel{r_{k}}{\longrightarrow} \mathbb{C}^{k \operatorname{dim} A}$. We first show that there is a positive integer $k$ such that

$$
r_{k} \circ j_{k}(f)(0) \notin r_{k}\left(\left.\operatorname{supp} D^{(k)}\right|_{J_{k}(A)}\right) \subset \mathbb{C}^{k \operatorname{dim} A} .
$$

Suppose that $r_{k} \circ j_{k}(f)(0) \in r_{k}\left(\left.\operatorname{supp} D^{(k)}\right|_{J_{N}(A)}\right)$ for all $k \geq 0$. Then we have

$$
\left.\operatorname{supp} D^{(k)}\right|_{J_{N}(A)} \cap r_{k}^{-1}\left(r_{k} \circ j_{k}(f)(0)\right) \neq \phi
$$

for all $k \geq 0$, thus we have

$$
V_{k} \stackrel{\text { def }}{=} p_{k}\left(\left.\operatorname{supp} D^{(k)}\right|_{J_{N}(A)} \cap r_{k}^{-1}\left(r_{k} \circ j_{k}(f)(0)\right)\right) \neq \phi
$$

for all $k \geq 0$, where $p_{k}$ is the projection $p_{k}: J_{k}(A) \rightarrow A$. Note that $V_{k}$ is Zariski closed, (this is because $p_{k}: J_{k}(A) \rightarrow A$ has a section $\mathrm{id}_{A} \times\left\{r_{k} \circ\right.$ $\left.j_{k}(f)(0)\right\}: A \hookrightarrow J_{k}(A)$, and $V_{k}$ is just pull back of $\left.\operatorname{supp} D^{(k)}\right|_{J_{N}(A)}$ by this section) and note that $V_{k} \supset V_{k+1}$. Thus we have the sequence of Zariski closed sets,

$$
D \supset V_{1} \supset V_{2} \supset V_{3} \supset \cdots
$$


that eventually stabilizes at the variety $V$. Since we are assuming $V_{k} \neq \phi$ for all $k$, we have $V \neq \phi$. Let $a \in V$, and translate holomorphic curve $f$ by $a-f(0)$ and put $\widetilde{f}(z)=f(z)+a-f(0)$. Then by construction of $\widetilde{f}$, we have

$$
\widetilde{f}(0)=a \quad \text { and }\left.\quad j_{k}(\widetilde{f})(0) \in \operatorname{supp} D^{(k)}\right|_{J_{N}(A)} .
$$

Observing the Taylor series, we get $\tilde{f}(\mathbb{C}) \subset \operatorname{supp} D$, and hence a contradiction since we are assuming $f$ to be non-degenerate.

Now, we can take $k>0$ such that $r_{k} \circ j_{k}(f)(0) \notin r_{k}\left(\left.\operatorname{supp} D^{(k)}\right|_{J_{N}(A)}\right)$. Since $A$ is projective, $r_{k}$ is a closed morphism, so $r_{k}\left(\left.\operatorname{supp} D^{(k)}\right|_{J_{N}(A)}\right)$ is Zariski closed. Thus we can take a divisor $H$ of $\mathbb{C}^{k \operatorname{dim} A}$ such that

$$
r_{k} \circ j_{k}(f)(0) \notin H \quad \text { and } \quad r_{k}\left(\left.\operatorname{supp} D^{(k)}\right|_{J_{N}(A)}\right) \subset H .
$$

Consider the pull back divisor $r_{k}^{*} H$ on $J_{k} A$. Then since we have $\left.\operatorname{supp} D^{(k)}\right|_{J_{N}(A)} \subset \operatorname{supp} r_{k}^{*} H$, the proof of Proposition 2.3 .2 (3) implies that there is a positive integer $n>0$ such that

$$
\left.D^{(k)}\right|_{J_{N}(A)} \subset n r_{k}^{*} H=r_{k}^{*}(n H) .
$$

Note that there is a morphism $\psi^{\prime}: \mathbb{C}^{k \operatorname{dim} A} \rightarrow \mathbb{C}$ such that $\psi^{\prime *}(0)=n H$. Hence the morphism

$$
\psi=\operatorname{id}_{A} \times \psi^{\prime}: J_{k}(A)=A \times \mathbb{C}^{k \operatorname{dim} A} \longrightarrow A \times \mathbb{C}=K_{A}^{\vee}
$$

satisfies the condition $\left.D^{(k)}\right|_{J_{N}(A)} \subset \psi^{*} F_{0}^{\left[K_{A}^{\vee}\right]}$ and $j_{k}(f)(\mathbb{C}) \not \subset \psi^{*} F_{0}^{\left[K_{A}^{\vee}\right]}$. By Proposition 4.2.1, we have

$$
m(r, f, D)+N{ }_{\text {“Ram" } f}(r) \leq S(r, f) .
$$

Since $N$ "Ram" $f(r) \geq 0$, we have Theorem 4.3.1.

Remark 4.3.2. If we consider a holomorphic curve $f: Y \rightarrow A$, where $\pi: Y \rightarrow \mathbb{C}$ is a finite analytic covering space, we obtain the following statement;

Suppose $f(Y)$ is not contained in a translate of $D$. Then there is a positive constant $C$, depending on $f$ and $D$, such that

$$
m(r, f, D) \leq C N_{\pi, \operatorname{Ram}}(r)+S(r, f) .
$$


4.4. Next, we shall consider the Second Main Theorem for hyperplanes in the projective space $\mathbb{P}^{n}$, which is due to Cartan [C33].

TheOrem 4.4.1. Let $f: \mathbb{C} \rightarrow \mathbb{P}^{n}$ be a holomorphic map. Assume that the image of $f$ is not contained in any hyperplane. Let $H_{1}, \ldots, H_{s}$ be hyperplanes in general position. Then

$$
\sum_{k=1}^{s} m\left(r, f, H_{k}\right)+T\left(r, f, K_{\mathbb{P} n}\right)+N_{\operatorname{Ram} f}(r) \leq S(r, f) .
$$

Proof. First we shall observe that there is a morphism of fiber bundles over $\mathbb{P}^{n}$

$$
\psi: J_{n}\left(\mathbb{P}^{n}\right) \longrightarrow K_{\mathbb{P} n}^{\vee}
$$

such that

$$
\left.H_{1}^{(n)}\right|_{J_{n}\left(\mathbb{P}^{n}\right)}+\cdots+\left.H_{s}^{(n)}\right|_{J_{n}\left(\mathbb{P}^{n}\right)} \subset \psi^{*} F_{0}^{\left[K_{\mathbb{P}}^{\vee}\right]} .
$$

Let $\left(Z_{0}: \cdots: Z_{n}\right)$ be a system of homogeneous coordinates on $\mathbb{P}^{n}$. Then for $0 \leq i \leq n, U_{i}=\mathbb{P}^{n}-\left(Z_{i}=0\right) \simeq \mathbb{C}^{n}$ has the coordinates $\left(\frac{Z_{0}}{Z_{i}}, \ldots, \frac{Z_{i}}{Z_{i}}, \ldots, \frac{Z_{n}}{Z_{i}}\right)$. Moreover we have $J_{n}\left(U_{i}\right) \simeq \mathbb{C}^{n} \times \mathbb{C}^{n^{2}}$ and $T\left(U_{i}\right) \simeq \mathbb{C}^{n} \times \mathbb{C}^{n}$. For $1 \leq j \leq n$, consider the morphism

$$
\iota_{i, j}: J_{n}\left(U_{i}\right) \stackrel{\left(\frac{Z_{0}}{Z_{i}}, \ldots, \frac{V_{i}}{Z_{i}}, \ldots, \frac{Z_{n}}{Z_{i}}, d^{j} \frac{Z_{0}}{Z_{i}}, \ldots, d^{j} \frac{Z_{i}}{Z_{i}}, \ldots, d^{j} \frac{Z_{n}}{Z_{i}}\right)}{\longrightarrow} T\left(U_{i}\right) .
$$

Then we have the isomorphism $\iota_{i}=\iota_{i, 1} \times \cdots \times \iota_{i, n}$ of fiber bundles over $U_{i}$

$$
\iota_{i}: J_{n}\left(U_{i}\right) \stackrel{\sim}{\longrightarrow} \underbrace{T\left(U_{i}\right) \times_{U_{i}} \cdots \times_{U_{i}} T\left(U_{i}\right)}_{n \text { times }} .
$$

Under this isomorphism $\iota_{i}$, we have

$$
\left.\left.\iota_{i}^{*} H^{(1), n}\right|_{J_{n}\left(U_{i}\right)} \simeq H^{(n)}\right|_{J_{n}\left(U_{i}\right)}
$$

for a hyperplane $H \subset \mathbb{P}^{n}$. Next consider the morphism of fiber bundles

$$
\psi_{i}=\tau_{\wedge} \circ \iota_{i}: J_{n}\left(U_{i}\right) \longrightarrow K_{U_{i}}^{\vee} \simeq\left\{U_{i}\right\} \times \mathbb{C}
$$


Set $\omega_{i}=d \frac{Z_{0}}{Z_{i}} \wedge \cdots \wedge d \frac{\vee}{Z_{i}} \wedge \cdots \wedge d \frac{Z_{n}}{Z_{i}}$. Then $K_{U_{i}}^{\vee}$ has coordinates $\left(u, \omega_{i}\right)$ where $u_{K_{U_{i}}^{\vee}}^{\vee}: K_{U_{i}}^{\vee} \rightarrow U_{i}$ is the projection. Now we shall show $\psi_{i}=\psi_{j}$ on $U_{i} \cap U_{j}$ to obtain $\psi: J_{n}\left(\mathbb{P}^{n}\right) \rightarrow K_{\mathbb{P}^{n}}^{\vee}$. Since $\omega_{i}=(-1)^{i-j} \frac{Z_{j}^{n+1}}{Z_{i}^{n+1}} \omega_{j}$ on $U_{i} \cap U_{j}$, and

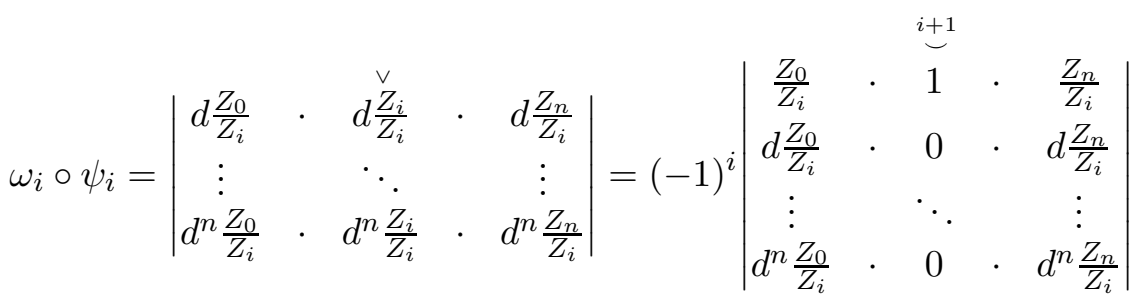

$$
\begin{aligned}
& =(-1)^{i} \frac{1}{Z_{i}^{n+1}}\left|\begin{array}{ccc}
Z_{0} & \cdots & Z_{n} \\
\vdots & \ddots & \vdots \\
d^{n} Z_{0} & \cdots & d^{n} Z_{n}
\end{array}\right|=(-1)^{i-j} \frac{Z_{j}^{n+1}}{Z_{i}^{n+1}} \omega_{j} \circ \psi_{j},
\end{aligned}
$$

we have $\psi_{i}=\psi_{j}$. Gluing $\psi_{i}$ 's, we obtain $\psi: J_{n}\left(\mathbb{P}^{n}\right) \rightarrow K_{\mathbb{P}^{n}}^{\vee}$. Consider the divisor $\psi^{*} F_{0}^{\left[K_{\mathbb{P} n}^{\vee}\right]}$. From Lemma 4.1.2 and (4.4.3), we have (4.4.2). Moreover for a holomorphic curve $f: \mathbb{C} \rightarrow \mathbb{P}^{n}$, we have

$$
f(\mathbb{C}) \subset \psi^{*} F_{0}^{\left[K_{\mathbb{P} n}^{\vee}\right]} \Longleftrightarrow f(\mathbb{C}) \subset H \text { for some hyperplane } H \subset \mathbb{P}^{n} .
$$

Now consider a holomorphic curve $f: \mathbb{C} \rightarrow \mathbb{P}^{n}$ such that the image of $f$ is not contained in any hyperplane. Then we have $f(\mathbb{C}) \not \subset \psi^{*} F_{0}^{\left[K_{\mathbb{P} n}^{\vee}\right]}$. By Proposition 4.2.1, we have

$$
\sum_{k=1}^{s} m\left(r, f, H_{k}\right)+T\left(r, f, K_{\mathbb{P}^{n}}\right)+N_{\text {“Ram" } f}(r) \leq S(r, f) .
$$

But by a straightforward computation, we can show that the term $N$ "Ram" $f(r)$ is a usual term $N_{\text {Ram } f}(r)$ such as Lang's book [L87]. Hence we have Theorem 4.4.1.

\section{$\S 5$. Extensions to the logarithmic jet space}

5.1. We shall extend our results to the Logarithmic jet space due to Noguchi [No86]. Let $V$ be a smooth quasi-projective variety. Let $D \subset V$ be a divisor with only simple normal crossings. For an integer $k \geq 0$, we can construct the Logarithmic $k$-th jet bundle $J_{k}(V ; \log D)$ along $D$, and a morphism

$$
\psi_{k}: J_{k}(V ; \log D) \longrightarrow J_{k}(V) .
$$


For $k \geq l$, we have a natural forgetful morphism

$$
p_{k, l}^{\log }: J_{k}(V ; \log D) \longrightarrow J_{l}(V ; \log D) .
$$

We have $J_{0}(V ; \log D)=V$, and $p_{k, 0}^{\log }: J_{k}(V ; \log D) \rightarrow V$ is the fiber bundle whose fibers are $\mathbb{C}^{k \operatorname{dim} V}$. In the following, we shall write $p_{k, 0}^{\log }$ simply as $p_{k}$. For $\phi \in \Gamma\left(V, \mathcal{O}_{V}\right)$ and an integer $l(0 \leq l \leq k)$, we have $d_{\log }^{l} \phi \in$ $\Gamma\left(J_{k}(V ; \log D), \mathcal{O}_{J_{k}(V ; \log D)}\right)$ by $d_{\log }^{l} \phi=d^{l} \phi \circ \psi_{k}$. Then $d_{\log }^{l}$ also satisfies the Leibniz rule $(2.4 .1),(2.4 .2)$. We have the following proposition.

Proposition 5.1.1. Let $V, D$ be as above. Suppose $V$ is affine. Let $\varphi_{1}, \ldots, \varphi_{d} \in \Gamma\left(V, \mathcal{O}_{V}\right)(d=\operatorname{dim} V)$ form a local coordinate system around every point of $V$, and let $D=\left(\varphi_{1} \cdots \varphi_{i}\right)$, i.e. $\varphi_{1} \cdots \varphi_{i}=0$ defines $D$. Then we have

$$
\begin{aligned}
& J_{k}(V ; \log D)=V \times \operatorname{Spec} \mathbb{C} {\left[\frac{d_{\log \varphi_{1}}}{\varphi_{1}}, \ldots, \frac{d_{\log \varphi_{i}}}{\varphi_{i}}, d_{\log } \varphi_{i+1}, \ldots, d_{\log } \varphi_{d},\right.} \\
&\left.\ldots, \frac{d_{\log }^{k} \varphi_{1}}{\varphi_{1}}, \ldots, \frac{d_{\log }^{k} \varphi_{i}}{\varphi_{i}}, d_{\log }^{k} \varphi_{i+1}, \ldots, d_{\log }^{k} \varphi_{d}\right] .
\end{aligned}
$$

Proof. See [No86, p. 234].

The sheaf of $\mathcal{O}_{V}$ algebra $p_{k *} \mathcal{O}_{J_{k}(V ; \log D)}$ is naturally the sheaf of graded algebra such that the weight of $d_{\log }^{l} \phi$ is $l$ and the subalgebra of weight 0 elements is $\mathcal{O}_{V}$. We have

$$
J_{k}(V ; \log D)=\operatorname{Spec} p_{k *} \mathcal{O}_{J_{k}(V ; \log D)}
$$

and we shall compactify $J_{k}(V ; \log D)$ by

$$
\bar{J}_{k}(V ; \log D)=\operatorname{Proj} p_{k *} \mathcal{O}_{J_{k}(V ; \log D)} \bigotimes_{\mathcal{O}_{V}} \mathbb{C}\left[\eta_{\infty}^{\log }\right]
$$

where the weight of $\eta_{\infty}^{\log }$ is 1. As in Section 2, we shall use the notations $d_{\log }^{l} \phi \otimes 1$ (we have $\left.d_{\log }^{l} \phi=\left(d_{\log }^{l} \phi \otimes 1\right) /\left(\eta_{\infty}^{\log }\right)^{l}\right)$ and $\partial J_{k}(V ; \log D)=\left(\eta_{\infty}^{\log }\right)$, which is a $\mathbb{Q}$-Cartier divisor on $\bar{J}_{k}(V ; \log D)$.

For a closed subscheme $Z \subset V$, we shall define the closed subscheme $Z_{\log }^{(k)} \subset \bar{J}_{k}(V ; \log D)$ in the following manner. If $V$ is affine and $\Gamma\left(V, \mathcal{I}_{Z}\right)$ is generated by $\phi_{1}, \ldots, \phi_{j} \in \Gamma\left(V, \mathcal{O}_{V}\right)$, then $Z_{\log }^{(k)}$ is defined by a homogeneous ideal

$$
\left(\phi_{1}, \ldots, \phi_{j}, d_{\log } \phi_{1} \otimes 1, \ldots, d_{\log } \phi_{j} \otimes 1, \ldots, d_{\log }^{k} \phi_{1} \otimes 1, \ldots, d_{\log }^{k} \phi_{j} \otimes 1\right)
$$


of $p_{k *} \mathcal{O}_{J_{k}(V ; \log D)} \bigotimes_{\mathcal{O}_{V}} \mathbb{C}\left[\eta_{\infty}^{\log }\right]$. In the case $V$ is general, take a Zariski open covering $\left\{U_{\epsilon}\right\}$ of $V$, and construct $\left(\left.Z\right|_{U_{\epsilon}}\right)_{\log }^{(k)}$. Then glue these $\left(\left.Z\right|_{U_{\epsilon}}\right)_{\log }^{(k)}$, s and define the subscheme $Z_{\log }^{(k)}$ of $\bar{J}_{k}(V ; \log D)$.

Next we shall state the logarithmic version of Proposition 3.1.1. Let $X$ be a smooth projective variety. Let $D$ be a divisor on $X$ with only simple normal crossings. Following condition (a)' for a set $A$ of rational functions on $X$ is a modification of the condition (a).

(a) ${ }^{\prime}$ For every point $P \in X$, there is a Zariski open neighborhood $U$ of $P$ and rational functions $\varphi_{1}, \ldots, \varphi_{d} \in A$ such that $\varphi_{1}, \ldots, \varphi_{d}$ are holomorphic on $U$ and form a local coordinate system around every point of $U$. Moreover $\left.D\right|_{U}$ is defined by $\varphi_{1} \cdots \varphi_{i}=0(0 \leq i \leq d)$. Here $d=\operatorname{dim} X$ and $i$ depends on $P$ and $U$.

Note that we can find a finite set $A$ of rational functions on $X$ which satisfies condition $(\mathrm{a})^{\prime}$.

Proposition 5.1.2. Let $X, D$ be as above. Let $Z \subset X$ be a closed subscheme. Suppose that $A$ and $B$ are finite sets of rational functions on $X$ satisfing the conditions $(\mathrm{a})^{\prime}$ and $(\mathrm{b})_{Z}$ respectively. Then we have

$$
\begin{aligned}
& \lambda_{\partial J_{l}(X ; \log D)} \leq \sum_{\varphi \in A} \sum_{1 \leq s \leq l} \log ^{+}\left|\frac{d_{\log }^{s} \varphi}{\varphi}\right|+O(1) \quad \text { on } \bar{J}_{l}(X ; \log D) . \\
& \lambda_{Z} \circ p_{l} \leq \lambda_{Z_{\log }^{(l)}}+\sum_{\varphi \in B} \sum_{1 \leq s \leq l} \log ^{+}\left|\frac{d_{\log }^{s} \varphi}{\varphi}\right|+O(1) \quad \text { on } \bar{J}_{l}(X ; \log D) .
\end{aligned}
$$

Proof. The proof of Proposition 5.1.2 (2) is completely analogous to that of Proposition 3.1.1 (2), hence we don't give any detail here.

The proof of Proposition 5.1.2 (1) is analogus to that of Proposition 3.1 .1 (1) except for some modifications. We now present the necessary modifications. For $P \in X$, we shall take its Zariski open neighborhood $U$ and rational functions $\varphi_{1}, \ldots, \varphi_{d} \in A$ such as condition (a)'. Then $\varphi_{1}, \ldots, \varphi_{d}$ are holomorphic on $U$ and $\varphi_{1} \cdots \varphi_{i}=0$ is the defining equation for $\left.D\right|_{U}$. By the straightforward modification of (3.1.2), we have

$$
\begin{array}{r}
\lambda_{\left(\eta_{\infty}^{\log }\right)} \leq\left\langle\bar{J}_{l}\left(U ; \log D||_{U}\right)\right\rangle \\
\min \left(\lambda_{V\left(\eta_{\infty}^{\log }\right)}, \lambda_{V\left(d_{\log }^{s} \varphi_{t} \otimes 1\right)}\right)+\log ^{+}\left|\frac{d_{\log }^{s} \varphi_{t}}{\varphi_{t}}\right| \\
(\text { for } i+1 \leq t \leq d, 1 \leq s \leq l) .
\end{array}
$$


Consider the tautological equality

$$
0=\min \left(0,-\log \left|\frac{d_{\log }^{s} \varphi_{t}}{\varphi_{t}}\right|\right)+\log \max \left(1,\left|\frac{d_{\log }^{s} \varphi_{t}}{\varphi_{t}}\right|\right)
$$

for $1 \leq s \leq l, 1 \leq t \leq i$. On the other hand, we have the equation

$$
\left(\frac{d_{\log }^{s} \varphi_{t}}{\varphi_{t}}\right)=\left(\frac{d_{\log \varphi_{t} \otimes 1}}{\varphi_{t}}\right)-s\left(\eta_{\infty}^{\log }\right)
$$

for the $\mathbb{Q}$-Cartier divisors on $\bar{J}_{l}\left(U ;\left.\log D\right|_{U}\right)$. Here note that since $d_{\log }^{s} \varphi_{t} / \varphi_{t}$ is holomorphic on $J_{l}\left(U ;\left.\log D\right|_{U}\right)$ for $1 \leq t \leq i$, we have $\left(d_{\log }^{s} \varphi_{t} \otimes 1\right) / \varphi_{t} \in$ $p_{k *} \mathcal{O}_{J_{k}\left(U ;\left.\log D\right|_{U}\right)} \otimes \mathbb{C}\left[\eta_{\infty}^{\log }\right]$ as weight $s$ element. By Lemma 2.2.11, (2.4.4), (5.1.4) and (5.1.5), we have

$$
0 \leq_{\left\langle\bar{J}_{l}\left(U ; \log D||_{U}\right)\right\rangle} \min \left(0, \lambda_{V\left(\frac{d_{\log \varphi}^{s} \varphi_{t} \otimes 1}{\varphi_{t}}\right)}-s \lambda_{\left(\eta_{\infty}^{\log }\right)}\right)+\log \max \left(1,\left|\frac{d_{\log \varphi_{t}}^{s}}{\varphi_{t}}\right|\right) .
$$

Hence we have

$$
\begin{array}{r}
\lambda_{\left(\eta_{\infty}^{\log }\right)} \leq\left\langle\bar{J}_{l}(U ; \log D \mid U)\right\rangle \\
\min \left(\lambda_{V\left(\eta_{\infty}^{\log }\right)}, \lambda_{V\left(\frac{d_{\log }^{s} \varphi_{t} \otimes 1}{\varphi_{t}}\right)}\right)+\log ^{+}\left|\frac{d_{\log }^{s} \varphi_{t}}{\varphi_{t}}\right| \\
(\text { for } 1 \leq t \leq i, 1 \leq s \leq l)
\end{array}
$$

By (5.1.3), (5.1.6) we have

$$
\begin{array}{r}
\lambda_{\left(\eta_{\infty}^{\log }\right)} \leq\left\langle\bar{J}_{l}\left(U ;\left.\log D\right|_{U}\right)\right\rangle \\
\min _{\substack{1 \leq s \leq l \\
1 \leq t \leq i \\
i+1 \leq u \leq d}}\left\{\lambda_{V\left(\eta_{\infty}^{\log }\right), \lambda_{V}\left(\frac{d_{\log }^{s} \varphi_{t} \otimes 1}{\varphi_{t}}\right)}, \lambda_{V\left(d^{s} \varphi_{u} \otimes 1\right)}\right\} \\
+\sum_{\varphi \in A} \sum_{1 \leq s \leq l} \log ^{+}\left|\frac{d_{\log }^{s} \varphi}{\varphi}\right| .
\end{array}
$$

Hence by Proposition 5.1.1, we have

$$
\lambda_{\left(\eta_{\infty}^{\log }\right)} \leq_{\left\langle\bar{J}_{l}(U ; \log D \mid U)\right\rangle} \sum_{\varphi \in A} \sum_{1 \leq s \leq l} \log ^{+}\left|\frac{d_{\log }^{s} \varphi}{\varphi}\right|,
$$

which implies Proposition 5.1.2 (1). 
Let $Y \stackrel{\pi}{\rightarrow} \mathbb{C}$ be a finite analytic covering space. Let $f: Y \rightarrow X$ be a holomorphic curve such that $f(Y) \not \subset \operatorname{supp} D$. Then there is a logarithmic $l$-th jet lifting

$$
j_{l}^{\log }(f): Y \longrightarrow \bar{J}_{l}(X ; \log D)
$$

such that $\psi_{l} \circ j_{l}^{\log }(f)=j_{l}(f)$. The following logarithmic version of Theorem 3.2.1 is obtained from Proposition 5.1.2.

TheOREM 5.1.7. (Logarithmic ANLD) Let $X$ be a smooth projective variety, and $D \subset X$ be a divisor with only simple normal crossings. Let $f: Y \rightarrow X$ be a holomorphic curve. Let $Z \subset X$ be a closed subscheme. Suppose $f(Y) \not \subset \operatorname{supp} Z \cup \operatorname{supp} D$. Then we have

$$
\begin{aligned}
& m(r, f, Z) \leq m\left(r, j_{l}^{\log }(f), Z_{\log }^{(l)}\right)+S(r, f) . \\
& m\left(r, j_{l}^{\log }(f), \partial J_{l}(X ; \log D)\right) \leq S(r, f) .
\end{aligned}
$$

Proof. The proof is completely analogous to that of Theorem 3.2.1.

Next we shall state the logarithmic version of Proposition 4.2.1.

Proposition 5.1.8. Let $X, D$ be as above. Let $E=E_{1}+\cdots+E_{s} \subset$ $X$ be a divisor. Suppose that there are a positive integer $N>0$, a line bundle $L \stackrel{u_{L}}{\longrightarrow} X$, and a morphism of fiber bundles $\varrho: J_{N}(X ; \log D) \rightarrow$ $L^{\vee}$ such that $\left.E_{1}^{(N)} \log \right|_{J_{N}(X ; \log D)}+\cdots+E_{s}^{(N)} \log \mid J_{N}(X ; \log D) \subset \varrho^{*} F_{0}^{\left[L^{\vee}\right]}$. Then for a holomorphic curve $f: \mathbb{C} \rightarrow X \backslash D$ with a non-degeneracy condition $j_{N}^{\log }(f)(\mathbb{C}) \not \subset \varrho^{*} F_{0}^{\left[L^{\vee}\right]}$, we have

$$
\sum_{i=1}^{s} m\left(r, f, E_{i}\right)+N_{\text {"Ram" } f}^{\log }(r)+T(r, f, L) \leq S(r, f) .
$$

Here we define the term $N_{\text {"Ram" } f}^{\log }(r)$ by $N_{\text {"Ram" } f}^{\log }(r)=N\left(r, \psi \circ j_{N}^{\log }(f), F_{0}^{\left[L^{\vee}\right]}\right)$.

Proof. The proof is analogous to that of Proposition 4.2.1. We just note the following fact. If we have a holomorphic curve $f: \mathbb{C} \rightarrow X \backslash D$, i.e. a holomorphic curve $f: \mathbb{C} \rightarrow X$ omitting the divisor $D$, then we have $N\left(r, j_{N}^{\log }(f), \partial J_{N}(X ; D)\right)=0$, and $N\left(r, \varrho \circ j_{N}^{\log }(f), F_{\infty}^{\left[L^{\vee}\right]}\right)=0$. Hence by Theorem 5.1.7 (2), we see

$$
T\left(r, \varrho \circ j_{N}^{\log }(f), F_{\infty}^{\left[L^{\vee}\right]}\right)=m\left(r, \varrho \circ j_{N}^{\log }(f), F_{\infty}^{\left[L^{\vee}\right]}\right) \leq S(r, f) .
$$


The following corollary is the logarithmic version of Corollary 4.2.3. Cf. [SiY97].

Corollary 5.1.9. Let $X, D$ be as above. Let $E \subset X$ be an ample divisor. Suppose there is a positive integer $k$ and a meromorphic $k$ jet differential $\omega$ on $X$ of at most log-pole singularity along $D$ (i.e. $\omega \in$ $\left.\Gamma\left(X, p_{k, 0 *}^{\log } \mathcal{O}_{J_{k}(X ; D)}\right)\right)$ such that $\omega$ vanishes on $E$ but is not identically zero on $X$. Then for any holomorphic curve $f: \mathbb{C} \rightarrow X \backslash D$ the pullback $f^{*} \omega$ is identically zero on $\mathbb{C}$.

Proof. The proof is analogous to that of Corollary 4.2.3. Using the notations of the proof of Corollary 4.2.3, we just give the necessary modifications. Suppose $f^{*} \omega$ is not identically zero on $\mathbb{C}$. Then since $f$ is rational, we have $f(\mathbb{C})=\phi\left(\mathbb{P}^{1} \backslash\{\infty\}\right)$. Hence $\phi\left(\mathbb{P}^{1} \backslash\{\infty\}\right)$ doesn't intersect $D$, and we have the morphism $J_{k}\left(\mathbb{P}^{1} ; \infty\right) \rightarrow J_{k}(X ; D)$.

5.2. We shall apply our logarithmic ANLD to the second main theorem for holomorphic curves in semi-Abelian varieties. We shall use [NoWY02] for the standard reference for this subsection. Let $A$ be a semiAbelian variety, and $\bar{A}$ be its natural compactification (cf. [NoWY02, p. 9]). Put $\partial A=\bar{A}-A$. Let

$$
\partial A=\bigcup_{j=1}^{p} B_{j}
$$

be the Whitney stratification of the divisor $\partial A$; that is, $B_{j}$ consists of all points $x \in \partial A$ such that the number of irreducible components of $\partial A$ passing $x$ is exactly $j$. Let $D \subset \bar{A}$ be a divisor. We shall consider the following boundary condition for $D$ (cf. [NoWY02, Condition 4.11]).

(BC) $D$ does not contain any stratum of $B_{p}$.

Note that the strata of $B_{p}$ are minimal.

Then the following Theorem is established in [NoWY02]. Here we shall discuss in the context of our logarithmic ANLD.

TheOrem 5.2.1. Let $A$ be a semi-Abelian variety, and $\bar{A}$ be its natural compactification. Let $D \subset \bar{A}$ be a divisor. Assume that $D$ satisfies the boundary condition (BC). Let $f: \mathbb{C} \rightarrow A$ be a holomorphic curve such that the image $f(\mathbb{C})$ is Zariski dense in $A$. Then we have

$$
m(r, f, D) \leq S(r, f) .
$$


Proof. The proof is analogous to that of Theorem 4.3.1, and we only sketch the necessary modifications. We shall show that there is a positive integer $N$, and a morphism of fiber bundles $\phi: J_{N}(\bar{A} ; \log \partial A) \rightarrow K_{\bar{A}}(\partial A)^{\vee}$

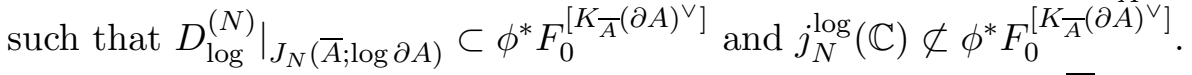

By the flat structure of the logarithmic tangent bundle $T(\bar{A} ; \log \partial A)$, we have

$$
J_{k}(\bar{A} ; \log \partial A) \simeq \bar{A} \times \mathbb{C}^{\operatorname{dim} A k},
$$

i.e. a trivialization of the logarithmic jet bundle over $\bar{A}$ (cf. [No86, p. 233]). Let $r_{k}: J_{k}(\bar{A} ; \log \partial A) \rightarrow \mathbb{C}^{\operatorname{dim} A k}$ be the projection. Then some complicated modification of the corresponding argument in the proof of Theorem 4.3.1 gives the following statement.

If $D$ satisfies the boundary condition (BC), then there is a positive integer $N_{0}$ such that

$$
r_{N_{0}} \circ j_{N_{0}}^{\log }(f)(0) \notin r_{N_{0}}\left(\left.\operatorname{supp} D_{\log }^{\left(N_{0}\right)}\right|_{J_{N_{0}}(\bar{A} ; \log \partial A)}\right) \subset \mathbb{C}^{k \operatorname{dim} A} .
$$

For the proof of this statement, see [NoWY02, Lemma 5.4]. Then putting $N=N_{0}$, we have a morphism of fiber bundles

$$
\phi: J_{N}(\bar{A} ; \log \partial A) \longrightarrow K_{\bar{A}}(\partial A)^{\vee} \simeq \bar{A} \times \mathbb{C}
$$

such that $\left.D_{\log }^{(N)}\right|_{J_{N}(\bar{A} ; \log \partial A)} \subset \phi^{*} F_{0}^{\left[K_{\bar{A}}(\partial A)^{\vee}\right]}$ and $j_{N}^{\log }(\mathbb{C}) \not \subset \phi^{*} F_{0}^{\left[K_{\bar{A}}(\partial A)^{\vee}\right]}$. Hence Proposition 5.1.8 implies our theorem.

\section{REFERENCES}

[B26] A. Bloch, Sur les systemes de fonctions uniformes satisfaisant a l'équation d'une variété algébrique dont l'irrégularité dépasse la dimension, J. Math. Pures Appl., 5 (1926), 9-66.

[CG72] J. Carlson and P. Griffiths, A defect relation for equidimensional holomorphic mappings between algebraic varieties, Ann. Math., 95 (1972), 557-584.

[C33] H. Cartan, Sur les zéros des combinaisons linéaires de p fonctions holomorphes données, Mathematica, 7 (1933), 5-31.

[GG80] M. Green and P. Griffiths, Two applications of algebraic geometry to entire holomorphic mappings, The Chern Symposium 1979, Springer-Verlag, New York-Heidelberg-Berlin (1980), pp. 41-74.

[GH78] P. Griffiths and J. Harris, Principles of Algebraic Geometry, John Wiley and Sons, 1978.

[GK73] P. Griffiths and J. King, Nevanlinna theory and holomorphic mappings between algebraic varieties, Acta Math., 130 (1973), 145-220. 
[H77] R. Hartshorne, Algebraic geometry, Springer-Verlag, Berlin, 1977.

[Kob91a] R. Kobayashi, Holomorphic curves in Abelian varieties, preprint (1991).

[Kob91b] R. Kobayashi, Holomorphic curves into algebraic subvarieties of an abelian variety, Internat. J. Math., 2 (1991), 711-724.

[Kob96a] R. Kobayashi, Restructuring value distribution theory, Geometric complex analysis (Hayama, 1995), World Sci. Publishing, River Edge, NJ (1996), pp. 37-354.

[Kob96b] R. Kobayashi, Nevanlinna theory and number theory, Sugaku, 48 (1996), no. 2, 113-127, (Japanese).

[Kob00] R. Kobayashi, Holomorphic curves in Abelian varieties: The second main theorem and applications, Japan. J. Math., 26 (2000), no. 1, 129-152.

[L87] S. Lang, Introduction to Complex Hyperbolic Spaces, Springer-Verlag, New York-Berlin-Heidelberg, 1987.

[M96] M. McQuillan, A Dynamical Counterpart to Faltings" "Diophantine Approximation on Abelian Varieties", preprint (1996).

[Nev39] R. Nevanlinna, Le théoreme de Picard-Borel et la théorie des fonctions méromophes, Gautheir-Villars, Paris, 1939.

[No76] J. Noguchi, Meromorphic mappings of a covering space over $\mathbb{C}^{m}$ into a projective variety and defect relations, Hiroshima Math. J., 6 (1976), 265-280.

[No77] J. Noguchi, Holomorphic curves in algebraic varieties, Hiroshima Math. J., 7 (1977), 833-853.

[No81] J. Noguchi, Lemma on logarithmic derivatives and holomorphic curves in algebraic varieties, Nagoya Math. J., 83 (1981), 213-233.

[No85] J. Noguchi, On the value distribution of meromorphic mappings of covering spaces over $\mathbb{C}^{m}$ into algebraic varieties, J. Math. Soc. Japan, 37 (1985), 295-313.

[No86] J. Noguchi, Logarithmic jet spaces and extensions of de Franchis' theorem, Contributions to Several Complex Variables, Aspects Math. No. 9, Vieweg, Braunschweig (1986), pp. 227-249.

[No98] J. Noguchi, On holomorphic curves in semi-Abelian varieties, Math. Z., 228 (1998), no. 4, 713-721.

[NoO90] J. Noguchi and T. Ochiai, Geometric function theory in several complex variables, Transl. Math. Mon. 80, Amer. Math. Soc., Providence, R.I., 1990.

[NoWY02] J. Noguchi, J. Winkelmann and K. Yamanoi, The second main theorem for holomorphic curves into semi-Abelian varieties, Acta Math., 188 (2002), no. 1, 129-161.

[O] T. Ochiai, On holomorphic curves in algebraic varieties with ample irregularity, Invent. Math., 43 (1977), 83-96.

[Sh75] B. Shiffman, Nevanlinna defect relations for singular divisors, Invent. Math., 31 (1975), 155-182.

[Sil87] J. Silverman, Arithmetic distance functions and height functions in diophantine geometry, Math. Ann., 279 (1987), no. 2, 193-216.

[SiY96] Y.-T. Siu and S.-K. Yeung, A generalized Bloch's theorem and the hyperbolicity of the complement of an ample divisor in an Abelian variety, Math. Ann., 306 (1996), 743-758.

[SiY97] Y.-T. Siu and S.-K. Yeung, Defects for ample divisors of Abelian varieties, 
Schwarz lemma, and hyperbolic hypersurfaces of low degrees, Amer. J. Math., 119 (1997), 1139-1172.

[Vi77] A. L. Vitter, The lemma of the logarithmic derivative in several complex variables, Duke Math. J., 44 (1977), 89-104.

Research Institute for Mathematical Sciences

Kyoto University

Oiwake-cho, Sakyo-ku

Kyoto, 606-8502

Japan

ya@kurims.kyoto-u.ac.jp 